\title{
Unsupervised Ensemble Kalman Filtering with an Uncertain Constraint for Land Hydrological Data Assimilation
}

\author{
M. Khaki ${ }^{\mathrm{a}, 1}$, B. Ait-El-Fquih ${ }^{\mathrm{b}}$, I. Hoteit ${ }^{\mathrm{b}}$, E. Forootan $^{\mathrm{c}}$, J. Awange $^{\mathrm{a}}$, M. Kuhn $^{\mathrm{a}}$ \\ ${ }^{a}$ School of Earth and Planetary Sciences, Discipline of Spatial Sciences, Curtin University, Perth, Australia. \\ ${ }^{b}$ King Abdullah University of Science and Technology (KAUST), Thuwal, Saudi Arabia. \\ ${ }^{c}$ School of Earth and Ocean Sciences, Cardiff University, Cardiff, UK.
}

\begin{abstract}
The standard ensemble data assimilation schemes often violate the dynamical balances of hydroand water flux changes. The present study aims at extending the recently introduced Weak Constrained Ensemble Kalman Filter (WCEnKF) to a more general framework, namely unsupervised WCEnKF (UWCEnKF), in which the covariance of the water balance model is no longer known, 6 thus requiring its estimation along with the model state variables. This extension is introduced because WCEnKF was found to be strongly sensitive to the (manual) choice of this covariance. The proposed UWCEnKF, on the other hand, provides a more general unsupervised framework that does not impose any (manual, thus heuristic) value of this covariance, but suggests an estimation of it, from the observations, along with the state. The new approach is tested based on numerical experiments of assimilating Terrestrial Water Storage (TWS) from Gravity Recovery and Climate Experiment (GRACE) and remotely sensed soil moisture data into a hydrological model. The experiments are conducted over different river basins, comparing WCEnKF, UWCEnKF, and the standard EnKF. In this setup, the UWCEnKF constrains the system state variables with TWS changes, precipitation, evaporation, and discharge data to balance the summation of water storage simulations. In-situ groundwater and soil moisture measurements are used to validate the results of the UWCEnKF and to evaluate its performances against the EnKF. Our numerical results clearly suggest that the proposed framework provides more accurate estimates of groundwater storage changes and soil moisture than WCEnKF and EnKF over the different studied basins.
\end{abstract}

Keywords: Constrained data assimilation, Ensemble Kalman Filter (EnKF), Unsupervised Weak Constrained Ensemble Kalman Filter (UWCEnKF), Water budget closure, Hydrological modeling.

Email address: Mehdi.Khaki@postgrad.curtin.edu.au (M. Khaki)

${ }^{1}$ Contact details: Department of Spatial Sciences, Curtin University, Perth, Australia, Email: Mehdi.Khaki@postgrad.curtin.edu.au, Tel: 0061410620379 


\section{Introduction}

Hydrological models play important roles in environmental studies and are crucial for hydrological applications. Due to a variety of factors, such as model structural errors, data deficiency, and uncertainty in inputs and parameters, the outputs of these models can be far from perfect. Data assimilation techniques offer a framework to improve the models simulations by constraining their outputs to the observations. However, the application of assimilation schemes could introduce an imbalance between water fluxes, namely precipitation $\mathbf{p}$, evaporation $\mathbf{e}$, discharge $\mathbf{q}$, and changes in water storage, $\Delta \mathbf{s}$, through the water balance equation $\Delta \mathbf{s}=\mathbf{p}-\mathbf{e}-\mathbf{q}$. The water balance equation is applied in land hydrological models to describe the relationships between these fluxes (Sokolov and Chapman, 1974). The model structure governs variations in the water state changes due to the incoming and outgoing hydrological water fluxes. Data assimilation of any water storages, e.g., soil moisture and/or terrestrial water storage (TWS), breaks the existing balance because the assimilated state does not satisfy the water balance property (Khaki et al., 2017a).

Existing data assimilation methodologies under water budget enforcement rely on a "perfect observations" assumption in the closure constraint (e.g., Pan and Wood, 2006; Sahoo et al., 2011; Pan et al., 2012). For example, Pan and Wood (2006) proposed a constrained ensemble Kalman filter $(\mathrm{CEnKF})$ that imposes regional water balance constraint to improve the filtering results. The CEnKF involves two successive EnKF-like updates. The first update uses the observations to update the state forecast, following an EnKF-like step, while the second update imposes the balance constraint via another EnKF-like correction, yet with a different form. Other studies have applied data merging algorithms along with the CEnKF (see, e.g., Sahoo et al., 2011; Pan et al., 2012; Zhang et al., 2016) to provide the flux datasets from various resources for water balance control. Although these improved datasets have resulted in better state estimates over different river basins by incorporating more accurate information about the constraints, the assumption of perfect observations is still problematic. This assumption leads to a strong constraint, which is unrealistic and may cause various issues. Simon and Chia (2002) suggested that even though it does not present any theoretical problems, the assumption can result in a singular covariance matrix, which in practice increases the possibility of numerical issues. Furthermore, by neglecting errors associated with flux observations, one can expect more estimation errors because of the strong water budget enforcement, which could also lead to over-fitting issues (Tangdamrongsub et al., 2017). 
In a recent study, Khaki et al. (2017a) proposed a new two-update ensemble Kalman-based scheme, a weak constrained ensemble Kalman filter (WCEnKF), that involves uncertainties in the water budget balance enforcement equation. Unlike previous studies (e.g., Pan and Wood, 2006; Sahoo et al., 2011; Pan et al., 2012; Khaki et al., 2017a), water balance uncertainty is added to the equality constraint formulation, which allows for a more realistic water balance control during filtering. This has been framed in a supervised framework, i.e., by assigning approximate error covariance to the water balance observations before filtering, which may not allow for an optimal estimation of corrections (in the second step of the filter) to be applied to results from the first step of the filter. The present study aims to extend the work of Khaki et al. (2017a) to the case where the covariance associated with flux observations is unknown, proposing an unsupervised framework to estimate it along with the hydrology state variable. The proposed Unsupervised WCEnKF (UWCEnKF) introduces an iterative scheme in the second update step of the WCEnKF.

In order to assess the performance of the UWCEnKF, numerical experiments are carried out to assimilate the Gravity Recovery And Climate Experiment (GRACE) derived terrestrial water storage (TWS), as well as soil moisture products from the Advanced Microwave Scanning Radiometer-Earth Observing System (AMSR-E) and Soil Moisture and Ocean Salinity (SMOS) into a hydrological model. Assimilating GRACE TWS data has been performed in a number of previous studies to constrain the mass balance of hydrological models over different river basins (e.g., Zaitchik et al., 2008; van Dijk et al., 2014; Eicker et al., 2014; Reager et al., 2015; Schumacher et al., 2016; Khaki et al., 2018a,b). Several studies already demonstrated a great capability of AMSR-E and SMOS datasets to constrain model estimates through data assimilation (e.g., De Jeu et al., 2008; Renzullo et al., 2014; Leroux et al., 2016; Tian et al., 2017). It has also been shown that simultaneous assimilation of the different datasets generally leads to better results in terms of state estimates (e.g., Zhang et al., 2014; Renzullo et al., 2014; Han et al., 2016; Tian et al., 2017; Lievens et al., 2017) as compared to individual assimilation of the different datasets. This motivates the current study to simultaneously assimilate GRACE TWS and soil moisture observations from AMSR-E and SMOS. We also apply the standard EnKF to compare its results with the proposed UWCEnKF filter. This enables to evaluate the relevance of the proposed approach for enforcing the water budget closure.

We further consider multiple observations of the water components in the water budget equation. This is done to achieve the best estimates of $\mathbf{p}$ and $\mathbf{e}$ over different basins (see Figure 1). Multi- 
mission products for precipitation and evaporation are used in the data merging approach of Sahoo et al. (2011) to derive a single data set for each observation type (i.e., p and e). The approach estimates uniform datasets independently for each basin. The merged data, as well as the water discharge measurements from various ground stations, are then applied to constrain the water balance equation in the UWCEnKF's second update. This experiment is undertaken over eight globally distributed basins; Amazon, Indus, Mississippi, Orange, Danube, St. Lawrence, MurrayDarling, and the Yangtze, to better explore the capability of the proposed filter.

The remainder of the paper is organized as follows. We first describe the data and model in Section 2. The UWCEnKF algorithm and experiments set up are described in Sections 3 and 4, respectively. We illustrate and discuss the experiments results in Section 5 and conclude the study in Section 6.

\section{Model and data}

\subsection{Hydrological model}

Vertical water compartments of the globally distributed World-Wide Water Resources Assessment system (W3RA) model, developed in 2008 by the Commonwealth Scientific and Industrial Research Organisation (CSIRO; Australia), are used to simulate water storages. W3RA is a onedimensional system that simulates landscape water stored in the vegetation and soil systems (van Dijk, 2010). Here, we use the $1^{\circ} \times 1^{\circ}$ version of the model to represent the water balance of the soil, groundwater and surface water storage, in which each cell is modeled independently from its neighbors (van Dijk, 2010). Groundwater dynamics in the model includes recharge from deep drainage, capillary rise (estimated with a linear diffusion equation), evaporation from groundwater saturated areas, and discharge. The model assumes that redistribution between grid cells can be ignored. Groundwater and river water dynamics are simulated at grid cell level and hence parameters are equal across the grid cell. Meteorological data sets of minimum and maximum temperature, downwelling short-wave radiation, and precipitation products provided by Princeton University (http://hydrology.princeton.edu) are used to force the W3RA model between 2003 and 2013. The model state is composed of the top, shallow and deep root soil water, snow, vegetation, groundwater, and surface water storage. 


\subsection{Assimilated observations}

Observations are assimilated in two steps. The first step assimilates GRACE TWS and satellite soil moisture observations, which are used to update the forecast state, while the second step enforces the water balance constraints, based on water flux observations.

\subsubsection{Data used in the first update}

GRACE level 2 (L2) gravity field data provided by the ITSG-Grace2016 (Mayer-Gürr et al., 2014 ) is used to compute monthly TWS after applying a few standard corrections. These include replacing degree 1 (C10, C11, S11) and degree 2 (C20) coefficients by more accurate coefficients from Swenson et al. (2008) and the Satellite Laser Ranging solutions (Cheng and Tapley, 2004), respectively. The gravity fields are then converted to $3^{\circ} \times 3^{\circ}$ TWS fields (Wahr et al., 1998). Khaki et al. (2017b) showed that implementing GRACE TWS with this spatial resolution exploits better impacts of GRACE TWS mainly because of larger correlation errors in the higher spatial resolution fields, which can be problematic during assimilation (see also Eicker et al., 2014; Schumacher et al., 2016). Colored/correlated noise and leakage errors are reduced using the Kernel Fourier Integration (KeFIn) filter, as proposed by Khaki et al. (2018c). The KeFIn filter works through a two-step post-processing algorithm: in the first step it mitigates the measurement noise and the aliasing of unmodelled high-frequency mass variations, and in the second step it decreases the leakage errors. Note that, here, rather using model outputs, fixed signal to noise ratio is applied during the KeFIn filtering (see Khaki et al., 2018c, for details). The application of the KeFIn filter was shown in Khaki et al. (2018c) to outperform a number of existing GRACE filtering techniques, e.g., landgrid-scaling method applied in Mass Concentration blocks (Mascons) products justifying its use in the current study.

Furthermore, soil moisture products from the Advanced Microwave Scanning Radiometer for EOS (AMSR-E) and ESA's Soil Moisture Ocean Salinity (SMOS) Earth Explorer mission are used to update soil storage variations. AMSR-E measures surface brightness temperature that corresponds to surface soil moisture content of $2 \mathrm{~cm}$ depth (Njoku et al., 2003). SMOS, on the other hand, measures microwave emissions from Earth's surface at about $5 \mathrm{~cm}$ depth. Here we use descending passes (see, e.g., De Jeu and Owe, 2003) of gridded Level-3 land surface product AMSR-E (Njoku, 2004) between 2003 and 2011, and Level 3 CATDS (Centre Aval de Traitement des Donnees SMOS) on ascending passes (see, e.g., Draper et al., 2009) for the period of 2011 
to 2013. These passes are selected due to their higher agreement with in-situ measurements (see also Jackson and Bindlish, 2012; Su et al., 2013). Both data products are rescaled to a monthly $1^{\circ} \times 1^{\circ}$ scale for the present study. Cumulative distribution function (CDF) matching (Reichle and Koster, 2004; Drusch et al., 2005) is applied to rescale the observations and remove the bias between the model simulations and observations. These measurements are mainly used to constrain the model variability, and not its absolute values. CDF matching relies on the assumption that the difference between observed soil moisture and that of the model is stationary and guarantees that the statistical distribution of both time series is the same (Draper et al., 2009; Renzullo et al., 2014).

\subsubsection{Data used in the second update}

Multiple data sets are used for flux net observations. Details of these products are outlined in Table 1. For precipitation, we use the Tropical Rainfall Measuring Mission (TRMM-3B43; Huffman et al., 2007), NOAA CPC Morphing Technique (CMORPH; Joyce et al., 2004), the Global Precipitation Climatology Project (GPCP) Version 2.3 (Adler et al., 2003), Global Precipitation Climatology Centre (GPCC; Schneider et al., 2008), and CPC unified gauge dataset (Chen et al., 2002). TRMM-3B43, CMORPH, and GPCP are used to generate the merged precipitation for data assimilation, while GPCC and CPC are applied for uncertainty analysis (cf. Section 4.1). Evaporation data are collected from MODIS Global Evapotranspiration Project (MOD16; Mu et al., 2007), Global Land Evaporation Amsterdam Model (GLEAM; Miralles et al., 2011), ERAinterim (Simmons et al., 2007), and Variable Infiltration Capacity (VIC) land surface model (Liang et al., 1994). Similar to precipitation, an uncertainty analysis is undertaken for evaporation with respect to ERA-interim and VIC products. All of these products are rescaled into a monthly $1^{\circ} \times 1^{\circ}$ spatial resolution. Various data sources are considered for discharge (see Table 1) to achieve the maximum amount of coverage within the basins of Amazon, Indus, Mississippi, Orange, Danube, St. Lawrence, Murray-Darling, and Yangtze (Figure 1).

FIGURE 1 


\subsection{In-situ measurements}

Monthly in-situ groundwater and soil moisture measurements are used to validate the results. The groundwater stations are located in the Mississippi, St. Lawrence, and Murray-Darling basins. Specific yield values provided by the literature (e.g., Gutentag et al., 1984; Strassberg et al., 2007; Seoane et al., 2013; Khaki et al., 2017a) are used to convert well measurements into groundwater storage anomalies. We further use in-situ soil moisture measurements over the Mississippi, St. Lawrence, Danube, Yangtze, and Murray-Darling basins to assess the estimated soil moisture. These data are collected from the International Soil Moisture Network (ISMN) and the moisturemonitoring network. It is worth mentioning that the temporal averages from the in-situ time series are removed before using them to validate the assimilation results. The distribution of both groundwater and soil moisture in-situ products are displayed in Figure 1. Details of the datasets are outlined in Table 1.

TABLE 1

\section{Methodology}

\subsection{Problem formulation}

Our discrete-time state-space system is represented as,

$$
\left\{\begin{array}{lll}
\mathbf{x}_{t} & = & \mathcal{M}_{t-1}\left(\mathbf{x}_{t-1}\right)+\nu_{t} \\
\mathbf{y}_{t} & = & \mathbf{H}_{t} \mathbf{x}_{t}+\mathbf{w}_{t}
\end{array}\right.
$$

where $\mathbf{x}_{t} \in \mathbb{R}^{n_{x}}$ and $\mathbf{y}_{t} \in \mathbb{R}^{n_{y}}$ stand for the system state and the observation at time $t$ and of sizes $n_{x}$ and $n_{y}$, respectively. In system $(1), \mathcal{M}_{t-1}($.$) is a nonlinear operator integrating the system state$ from time $t-1$ to $t$, and $\mathbf{H}_{t}$ is the observational (design) operator at time $t$, which is linear in our application. Note, however, that the proposed scheme can be easily extended to the nonlinear case (Liu and Xue, 2002). The model process noise, $\nu=\left\{\nu_{t}\right\}_{t=0}^{T}$, and the observation process noise, $\mathbf{w}=\left\{\mathbf{w}_{t}\right\}_{t=0}^{T}$, are assumed to be independent in time, jointly independent, and independent of the initial state, shown by $\mathbf{x}_{0}$. Furthermore, $\nu_{t}$ and $\mathbf{w}_{t}$ are assumed to be Gaussian with zero means and covariances $\mathbf{Q}_{t}$ and $\mathbf{R}_{t}$, respectively. The model time step, $t$, is considered to be equal to the 


\footnotetext{
${ }^{2}$ The term unconstrained comes from the fact that these pdfs are not based on the pseudo-observation, $\mathbf{z}_{t}$, that "represents" the equality constraint.
}

accounting for the uncertainty in the different water fluxes data through a noise term $\boldsymbol{\xi}_{t}$, which we assume here to be Gaussian with zero mean and covariance, $\boldsymbol{\Sigma}$, and independent of $\boldsymbol{\xi}_{t^{\prime} \neq t},\left\{\nu_{t}\right\}_{t=0}^{T}$, $\left\{\mathbf{w}_{t}\right\}_{t=0}^{T}$ and $\mathbf{x}_{0}$. Considering Eq. (2), one can see that changes in the water storage at two successive time steps is equal to the difference between precipitation and summation of evaporation and discharge up to uncertainties in the involved data. The constraint in Eq. (2) can be rewritten as another observation equation in the state-space formulation, Eq. (3), which also involves the state at the previous time,

$$
\mathbf{z}_{t}=\mathbf{G x}_{t}+\mathbf{L} \mathbf{x}_{t-1}+\boldsymbol{\xi}_{t}
$$

where $\mathbf{z}_{t} \stackrel{\text { def }}{=} \mathbf{d}_{t}-\mathbf{p}_{t}+\mathbf{e}_{t}+\mathbf{q}_{t}$ plays the role of a "pseudo-observation", $\mathbf{L}$ is an $n_{z} \times n_{x}$ identity matrix, and $\mathbf{G}=-\mathbf{L}$ (here, $n_{z}=n_{x}$ ). Define $\mathbf{r}_{t}=\left[\mathbf{y}_{t}^{T}, \mathbf{z}_{t}^{T}\right]^{T}$ and $\mathbf{r}_{0: t}=\left\{\mathbf{r}_{0}, \mathbf{r}_{1}, \cdots, \mathbf{r}_{t}\right\}$. In the state-space system (1)-(3), a generic filtering algorithm has been recently introduced by Khaki et al. (2017a), recursively computing the analysis pdf of the state $\mathbf{x}_{t}$ from the history of the augmented observations, $\mathbf{r}_{0: t}, p\left(\mathbf{x}_{t} \mid \mathbf{r}_{0: t}\right)$. The computation of $p\left(\mathbf{x}_{t} \mid \mathbf{r}_{0: t}\right)$ from $p\left(\mathbf{x}_{t-1} \mid \mathbf{r}_{0: t-1}\right)$ proceeds in a succession of a forecast step and two Bayesian update steps. The forecast step consists of moving from $p\left(\mathbf{x}_{t-1} \mid \mathbf{r}_{0: t-1}\right)$ to the forecast pdf, $p\left(\mathbf{x}_{t} \mid \mathbf{r}_{0: t-1}\right)$, based on the state transition pdf $p\left(\mathbf{x}_{t} \mid \mathbf{x}_{t-1}\right)$ (which is described by the state model). The resulting forecast pdf is then updated, based on the likelihood of the observations, $p\left(\mathbf{y}_{t} \mid \mathbf{x}_{t}\right)$ (which is represented by the observation model), resulting in an unconstrained analysis $\mathrm{pdf}^{2}, p\left(\mathbf{x}_{t} \mid \mathbf{r}_{0: t-1}, \mathbf{y}_{t}\right)$. The latter is, in turn, updated in the second Bayesian step, based on the likelihood of the pseudo-observation, $p\left(\mathbf{z}_{t} \mid \mathbf{x}_{t-1, t}\right)$ (which is represented by the constraint Eq. (3)), leading to the desirable analysis pdf at the current time $t, p\left(\mathbf{x}_{t} \mid \mathbf{r}_{0: t}\right)$. Details about these steps can be found in (Khaki et al., 2017a).

In a supervised framework, where the parameters of the constrained state-space system (includ- 
ing $\boldsymbol{\Sigma}$ ) are known, the above generic algorithm was implemented by Khaki et al. (2017a) through Monte Carlo approximation of the posterior mean (PM) estimate of the state and its covariance, which led to the ensemble Kalman-type WCEnKF. Khaki et al. (2017a) noticed that the WCEnKF is sensitive to the choice of $\boldsymbol{\Sigma}$, which can strongly affect the filter behaviors. Here, we design a more general unsupervised framework in which $\boldsymbol{\Sigma}$ is an unknown diagonal covariance matrix, which thereby needs to be estimated concurrently with the state.

\subsection{The Unsupervised Weak Constrained Ensemble Kalman Filter (UWCEnKF)}

\subsubsection{The generic algorithm}

The UWCEnKF shares the same forecast and first update steps as the WCEnKF, but computes the posterior distribution of both state and pseudo-observation noise covariance in the second update step, instead of only that of the state. In a Bayesian framework, this consists in viewing the covariance, $\boldsymbol{\Sigma}$, as another random variable with a given prior pdf; the goal is then to compute its posterior pdf jointly with the $\operatorname{state}^{3}, p\left(\mathbf{x}_{t-1}, \mathbf{x}_{t}, \boldsymbol{\Sigma} \mid \mathbf{r}_{0: t}\right)$. However, the statistical dependencies between the states, $\mathbf{x}_{t-1: t}$, and the covariance, $\boldsymbol{\Sigma}$, makes its computation quite tricky. One way to overcome this difficulty is to resort to the variational Bayesian (VB) approach and approximate $p\left(\mathbf{x}_{t-1}, \mathbf{x}_{t}, \boldsymbol{\Sigma} \mid \mathbf{r}_{0: t}\right)$ with a separable pdf $q\left(\mathbf{x}_{t-1}, \mathbf{x}_{t}, \boldsymbol{\Sigma} \mid \mathbf{r}_{0: t}\right)=q\left(\mathbf{x}_{t-1}, \mathbf{x}_{t} \mid \mathbf{r}_{0: t}\right) q\left(\boldsymbol{\Sigma} \mid \mathbf{r}_{0: t}\right)$, under the Kullback-Leibler divergence (KLD) minimization criteria (Jaakkola and Jordan, 2000; Smidl and Quinn, 2008; Ait-El-Fquih and Hoteit, 2015, 2016). This reads,

$$
\begin{aligned}
q\left(\mathbf{x}_{t-1}, \mathbf{x}_{t}, \mathbf{\Sigma} \mid \mathbf{r}_{0: t}\right) & =\underset{\phi\left(\mathbf{x}_{t-1}, \mathbf{x}_{t}, \boldsymbol{\Sigma} \mid \mathbf{r}_{0: t}\right)}{\operatorname{argmin}} \operatorname{KLD}\left(\phi\left(\mathbf{x}_{t-1}, \mathbf{x}_{t}, \mathbf{\Sigma} \mid \mathbf{r}_{0: t}\right)|| p\left(\mathbf{x}_{t-1}, \mathbf{x}_{t}, \mathbf{\Sigma} \mid \mathbf{r}_{0: t}\right)\right), \\
& =\underset{\phi\left(\mathbf{x}_{t-1}, \mathbf{x}_{t}, \boldsymbol{\Sigma} \mid \mathbf{r}_{0: t}\right)}{\operatorname{argmin}} \mathbb{E}_{\phi\left(\mathbf{x}_{t-1}, \mathbf{x}_{t}, \boldsymbol{\Sigma} \mid \mathbf{r}_{0: t}\right)}\left[\ln \left(\frac{\phi\left(\mathbf{x}_{t-1}, \mathbf{x}_{t}, \boldsymbol{\Sigma} \mid \mathbf{r}_{0: t}\right)}{p\left(\mathbf{x}_{t-1}, \mathbf{x}_{t}, \boldsymbol{\Sigma} \mid \mathbf{r}_{0: t}\right)}\right)\right],
\end{aligned}
$$

where $\mathbb{E}_{\phi(u)}[f(u)]$ denotes the expected value of $f(u)$ with respect to (w.r.t.) the pdf $\phi(u)$. The solution of Eq. (4) can be obtained from (the proof can be found for instance in Smidl and Quinn, 2006, pages 28-31):

$$
\begin{aligned}
q\left(\mathbf{x}_{t-1}, \mathbf{x}_{t} \mid \mathbf{r}_{0: t}\right) & \propto \exp \left(\mathbb{E}_{q\left(\boldsymbol{\Sigma} \mid \mathbf{r}_{0: t}\right)}\left[\ln \left(p\left(\mathbf{x}_{t-1}, \mathbf{x}_{t}, \boldsymbol{\Sigma}, \mathbf{r}_{0: t}\right)\right)\right]\right) \\
q\left(\boldsymbol{\Sigma} \mid \mathbf{r}_{0: t}\right) & \propto \exp \left(\mathbb{E}_{q\left(\mathbf{x}_{t-1}, \mathbf{x}_{t} \mid \mathbf{r}_{0: t}\right)}\left[\ln \left(p\left(\mathbf{x}_{t-1}, \mathbf{x}_{t}, \boldsymbol{\Sigma}, \mathbf{r}_{0: t}\right)\right)\right]\right) .
\end{aligned}
$$

\footnotetext{
${ }^{3}$ For the sake of clarity, the inclusion of both $\mathbf{x}_{t}$ and $\mathbf{x}_{t-1}$ in the joint posterior pdf of interest is due to the fact that both these states appear in the pseudo-observation model Eq. (3), which necessitates estimating both of them.
} 
According to Eqs. (5) and (6), the independence that is inserted between the marginal posteriors, $q\left(\mathbf{x}_{t-1}, \mathbf{x}_{t} \mid \mathbf{r}_{0: t}\right)$ and $q\left(\boldsymbol{\Sigma} \mid \mathbf{r}_{0: t}\right)$, is partially compensated by the fact that each of these pdfs remains dependent on the expected value of $\ln \left(p\left(\mathbf{x}_{t-1}, \mathbf{x}_{t}, \boldsymbol{\Sigma}, \mathbf{r}_{0: t}\right)\right)$ w.r.t. the other. However, this property of "cyclic" dependence between $q\left(\mathbf{x}_{t-1}, \mathbf{x}_{t} \mid \mathbf{r}_{0: t}\right)$ and $q\left(\boldsymbol{\Sigma} \mid \mathbf{r}_{0: t}\right)$ makes it impossible to exactly evaluate these pdfs, or any of their statistics, such as for instance their means, which are taken as the PM estimates of the states and the covariance, $\boldsymbol{\Sigma}$, respectively. A standard approximation is to proceed with cyclic iterations between (5) and (6), evaluating one pdf after the other, until convergence is reached (Smidl and Quinn, 2008; Sato, 2001; Massoud et al., 2018). Based on the factorization,

$$
p\left(\mathbf{x}_{t-1}, \mathbf{x}_{t}, \boldsymbol{\Sigma}, \mathbf{r}_{0: t}\right) \propto p\left(\mathbf{z}_{t} \mid \mathbf{x}_{t-1}, \mathbf{x}_{t}, \boldsymbol{\Sigma}\right) p\left(\mathbf{x}_{t-1}, \mathbf{x}_{t} \mid \mathbf{r}_{0: t-1}, \mathbf{y}_{t}\right) q\left(\boldsymbol{\Sigma} \mid \mathbf{r}_{0: t-1}\right),
$$

which stems from the conditional independence properties of the state-space system (1)-(3), the iterative form of Eqs. (5)-(6) becomes,

$$
\begin{aligned}
q^{(\ell)}\left(\mathbf{x}_{t-1}, \mathbf{x}_{t} \mid \mathbf{r}_{0: t}\right) & \propto \exp \left(\mathbb{E}_{q^{(\ell-1)}\left(\boldsymbol{\Sigma} \mid \mathbf{r}_{0: t}\right)}\left[\ln \left(p^{(\ell-1)}\left(\mathbf{z}_{t} \mid \mathbf{x}_{t-1}, \mathbf{x}_{t}, \boldsymbol{\Sigma}\right)\right)\right]\right) p\left(\mathbf{x}_{t-1}, \mathbf{x}_{t} \mid \mathbf{r}_{0: t-1}, \mathbf{y}_{t}\right), \\
q^{(\ell)}\left(\boldsymbol{\Sigma} \mid \mathbf{r}_{0: t}\right) & \propto \exp \left(\mathbb{E}_{q^{(\ell)}\left(\mathbf{x}_{t-1}, \mathbf{x}_{t} \mid \mathbf{r}_{0: t}\right)}\left[\ln \left(p^{(\ell-1)}\left(\mathbf{z}_{t} \mid \mathbf{x}_{t-1}, \mathbf{x}_{t}, \boldsymbol{\Sigma}\right)\right)\right]\right) q\left(\boldsymbol{\Sigma} \mid \mathbf{r}_{0: t-1}\right),
\end{aligned}
$$

where $p^{(\ell)}($.$) and q^{(\ell)}($.$) respectively denote the pdfs p($.$) and q($.$) at iteration \ell$. As can be seen below (cf. Section 3.2.2), iterating over the pdfs Eqs. (8)-(9) amounts in practice to iterate over their (approximate) parameters, thereby leading to an unsupervised ensemble-based filtering scheme, which iterates in its second step over the PM estimates of the states and the pseudo-observation noise covariance.

\subsubsection{Practical implementation}

For the sake of simplicity, we first focus on the case of a homogeneous noise with a covariance matrix,

$$
\boldsymbol{\Sigma}=\lambda \times \mathbb{I}_{n_{z}},
$$

where $\lambda$ is the variance value and $\mathbb{I}_{n_{z}}$ denotes the $n_{z} \times n_{z}$ identity matrix. The more general inhomogeneous case will be discussed later. The prior probability distribution $p(\lambda)$ is chosen as an inverse-Gamma distribution (as a natural choice for variances), with shape and scale parameters $\hat{\alpha}_{0}$ and $\hat{\beta}_{0}$, respectively (Smidl and Quinn, 2006). In the case of non-informative priors, one could take $\hat{\alpha}_{0}=\hat{\beta}_{0}$ relatively small. At each iteration $(\ell-1) \rightarrow(\ell)$, inserting in Eqs. (8) and (9) the 
Gaussian pdf,

$$
p^{(\ell-1)}\left(\mathbf{z}_{t} \mid \mathbf{x}_{t-1}, \mathbf{x}_{t}, \boldsymbol{\Sigma}\right)=\mathcal{N}_{\mathbf{z}_{t}}\left(\mathbf{G x}_{t}+\mathbf{L} \mathbf{x}_{t-1}, \boldsymbol{\Sigma}^{(\ell-1)}\right)
$$

one obtains a posterior $q^{(\ell)}\left(\lambda \mid \mathbf{r}_{0: t}\right)$ that is also an inverse-Gamma distribution with parameters, $\hat{\alpha}_{t}$ and $\hat{\beta}_{t}^{(\ell)}$, given in Eqs. (17)-(18) below. Likewise, $q^{(\ell)}\left(\mathbf{x}_{t-1}, \mathbf{x}_{t} \mid \mathbf{r}_{0: r}\right)$ is Gaussian with an ensemble representation given in Eqs. (14)-(16).

The $\boldsymbol{U} \boldsymbol{W} \boldsymbol{C E n K} \boldsymbol{F}$. Starting at time $t-1$ from an analysis ensemble, $\left\{\mathbf{x}_{t-1}^{a,(i)}\right\}_{i-1}^{m}$, and shape and scale parameters $\left(\hat{\alpha}_{t-1}, \hat{\beta}_{t-1}\right)$ of the inverse-Gamma posterior pdf $p\left(\lambda \mid \mathbf{r}_{0: t-1}\right)$, these at the next time $t$ can be computed following a succession of a forecast and two update steps. The forecast step, which computes the forecast ensemble, $\left\{\mathbf{x}_{t}^{f,(i)}\right\}_{i-1}^{m}$, and the first update step (with $\mathbf{y}_{t}$ ), which computes the unconstrained analysis and smoothing ensembles, $\left\{\tilde{\mathbf{x}}_{t}^{a,(i)}\right\}_{i-1}^{m}$ and $\left\{\tilde{\mathbf{x}}_{t-1}^{s,(i)}\right\}_{i-1}^{m}$, are identical to those in Khaki et al. (2017a), namely,

$$
\begin{aligned}
& \mathbf{x}_{t}^{f,(i)}=\mathcal{M}_{t-1}\left(\mathbf{x}_{t-1}^{a,(i)}\right)+\nu^{(i)}, \\
& \tilde{\mathbf{x}}_{t}^{a,(i)}=\mathbf{x}_{t}^{f,(i)}+\mathbf{P}_{\mathbf{x}_{t}^{f}} \mathbf{H}^{T} \underbrace{\left[\mathbf{H P}_{\mathbf{x}_{t}^{f}} \mathbf{H}^{T}+\mathbf{R}_{t}\right]^{-1}\left[\mathbf{y}_{t}+\epsilon^{(i)}-\mathbf{H} \mathbf{x}_{t}^{f,(i)}\right]}_{\mu_{t}^{(i)}}, \\
& \tilde{\mathbf{x}}_{t-1}^{s,(i)}=\mathbf{x}_{t-1}^{a,(i)}+\mathbf{P}_{\mathbf{x}_{t-1}^{a}, \mathbf{x}_{t}^{f}} \mathbf{H}^{T} \times \mu_{t}^{(i)},
\end{aligned}
$$

where $\mathbf{P}_{\mathbf{x}_{t}^{f}}$ is the sample forecast error covariance and $\mathbf{P}_{\mathbf{x}_{t-1}^{a}, \mathbf{x}_{t}^{f}}$ represents the sample cross-covariance between the previous analysis and current forecast errors, $\nu^{(i)} \sim \mathcal{N}\left(\mathbf{0}, \mathbf{Q}_{t}\right)$, and $\epsilon^{(i)} \sim \mathcal{N}\left(\mathbf{0}, \mathbf{R}_{t}\right)$.

As for the second update step (with $\mathbf{z}_{t}$ ), which applies the adjustment to enforce the water budget balance constraint, it involves iterations to compute Eqs. (8)-(9). Let $\hat{\alpha}_{t}=\hat{\alpha}_{t-1}+\frac{n_{z}}{2}$, the iteration begins with the initialization $\hat{\lambda}_{t}^{(0)}=\frac{\hat{\beta}_{t-1}}{\hat{\alpha}_{t}}$ and correspondingly $\hat{\boldsymbol{\Sigma}}_{t}^{(0)}=\hat{\lambda}_{t}^{(0)} \times \mathbb{I}_{n_{z}}$. For $\ell=0 \cdots L$, the state members are first updated as,

$$
\begin{aligned}
\mathbf{z}_{t}^{f,(i, \ell)} & =\mathbf{G} \tilde{\mathbf{x}}_{t}^{a,(i)}+\mathbf{L} \tilde{\mathbf{x}}_{t-1}^{s,(i)}+\boldsymbol{\xi}_{t}^{(i, \ell)} ; \quad \boldsymbol{\xi}_{t}^{(i, \ell)} \sim \mathcal{N}\left(\mathbf{0}, \hat{\mathbf{\Sigma}}_{t}^{(\ell)}\right), \quad i=1, \cdots, m, \\
\mathbf{x}_{t}^{a,(i, \ell)} & =\tilde{\mathbf{x}}_{t}^{a,(i)}+\mathbf{P}_{\tilde{\mathbf{x}}_{t}^{a}, \mathbf{z}_{t}^{f, \ell}} \underbrace{\left[\mathbf{M P} \mathbf{P}_{t} \mathbf{M}^{T}+\hat{\boldsymbol{\Sigma}}_{t}^{(\ell)}\right]^{-1}\left[\mathbf{z}_{t}-\mathbf{z}_{t}^{f,(i, \ell)}\right]}_{\nu_{t}^{(i, \ell)}}, \quad i=1, \cdots, m, \\
\mathbf{x}_{t-1}^{s,(i, \ell)} & =\tilde{\mathbf{x}}_{t-1}^{s,(i)}+\mathbf{P}_{\tilde{\mathbf{x}}_{t-1}^{s}, \mathbf{z}_{t}^{f, \ell}} \times \nu_{t}^{(i, \ell)}, \quad i=1, \cdots, m,
\end{aligned}
$$

where $\mathbf{M} \stackrel{\text { def }}{=}[\mathbf{G}, \mathbf{L}] ; \mathbf{P}_{\tilde{\mathbf{x}}_{t}^{a}, \mathbf{z}_{t}^{f, \ell}}$ and $\mathbf{P}_{\tilde{\mathbf{x}}_{t-1}^{s}, \mathbf{z}_{t}^{f, \ell}}$ are the sample cross-covariances computed using the 
ensembles $\left\{\tilde{\mathbf{x}}_{t}^{a,(i)}\right\}_{i=1}^{m},\left\{\tilde{\mathbf{x}}_{t-1}^{s,(i)}\right\}_{i=1}^{m}$ and $\left\{\mathbf{z}_{t}^{f,(i, \ell)}\right\}_{i=1}^{m}$; and $\mathbf{P}_{\boldsymbol{\eta}_{t}}$ is the sample covariance of the ensemble $\left\{\boldsymbol{\eta}_{t}^{(i)}\right\}_{i=1}^{m}$ with $\boldsymbol{\eta}_{t}^{(i)} \stackrel{\text { def }}{=}\left[\left(\tilde{\mathbf{x}}_{t}^{a,(i)}\right)^{T},\left(\tilde{\mathbf{x}}_{t-1}^{s,(i)}\right)^{T}\right]^{T}$. Based on the resulting ensembles, the observation noise variance is then updated as,

$$
\begin{aligned}
\hat{\beta}_{t}^{(\ell+1)} & =\hat{\beta}_{t-1}+\frac{1}{2}\left[\left\|\mathbf{z}_{t}-\mathbf{G} \hat{\mathbf{x}}_{t}^{a,(\ell)}-\mathbf{L} \hat{\mathbf{x}}_{t-1}^{s,(\ell)}\right\|^{2}+\operatorname{Trace}\left(\mathbf{M} \mathbf{P}_{\gamma_{t}^{\ell \ell}} \mathbf{M}^{T}\right)\right] \\
\hat{\lambda}_{t}^{(\ell+1)} & =\hat{\beta}_{t}^{(\ell+1)} / \hat{\alpha}_{t}, \\
\hat{\mathbf{\Sigma}}_{t}^{(\ell+1)} & =\hat{\lambda}_{t}^{(\ell+1)} \times \mathbb{I}_{n_{z}},
\end{aligned}
$$

where $\hat{\mathbf{x}}_{t}^{a,(\ell)}$ and $\hat{\mathbf{x}}_{t-1}^{s,(\ell)}$ are the (empirical) means of the ensembles $\left\{\mathbf{x}_{t}^{a,(i, \ell)}\right\}_{i=1}^{m}$ and $\left\{\mathbf{x}_{t-1}^{s,(i, \ell)}\right\}_{i=1}^{m}$, respectively; and $\mathbf{P}_{\boldsymbol{\gamma}_{t}^{\ell}}$ is the sample covariance of the ensemble $\left\{\boldsymbol{\gamma}_{t}^{(i, \ell)}\right\}_{i=1}^{m}$ with $\boldsymbol{\gamma}_{t}^{(i, \ell)} \stackrel{\text { def }}{=}\left[\left(\mathbf{x}_{t}^{a,(i, \ell)}\right)^{T},\left(\mathbf{x}_{t-1}^{s,(i, \ell)}\right)^{T}\right]^{T}$. The $\hat{\boldsymbol{\Sigma}}_{t}^{(L)}$ and $\left\{\mathbf{x}_{t}^{a,(i, L)}\right\}_{i=1}^{m}$ are then considered as the analysis covariance and state estimates, respectively, that will be used in the next assimilation cycle. In our numerical experiments, only few iterations (less than 10) were needed to reach convergence based on the variance estimate. Note that instead of pre-setting the number of iterations, $L$, on may use an alternative stopping criteria based, for instance, on the relative squared error norm (RSEN) of the estimated state and/or variance(s), or the evidence lower bound (ELB), defined as (Blei et al. , 2017),

$$
\mathcal{E}_{1}=\mathbb{E}_{q\left(\boldsymbol{\xi}_{t}, \boldsymbol{\Sigma} \mid \mathbf{r}_{0: t)}\right)}\left[\ln \left(p\left(\mathbf{v}_{t}, \boldsymbol{\Sigma}, \mathbf{r}_{t} \mid \mathbf{r}_{0: t-1}\right)\right)\right]-\mathbb{E}_{q\left(\boldsymbol{\xi}_{t}, \boldsymbol{\Sigma} \mid \mathbf{r}_{0: t}\right)}\left[\ln \left(q\left(\mathbf{v}_{t}, \boldsymbol{\Sigma} \mid \mathbf{r}_{0: t}\right)\right)\right]
$$

with $\mathbf{v}_{t}=\left[\mathbf{x}_{t}^{T}, \mathbf{x}_{t-1}\right]^{T}$. Note that it is not possible to use the KLD as this requires the knowledge of the target pdf, $p\left(\mathbf{v}_{t}, \mathbf{\Sigma} \mid \mathbf{r}_{0: t}\right)$, which, indeed, is not known. Furthermore, minimizing the KLD amounts to maximizing the ELB (Blei et al. , 2017). However, a problem occurs in practice with ELB (20) in case of large dimensional systems (i.e., when $n_{x}>m$ ). In such a case, the covariance $\mathbf{P}_{\gamma_{t}}$, whose inverse is involved in the expression of the (assumed Gaussian) pdf, $q\left(\mathbf{v}_{t} \mid \mathbf{r}_{0: t}\right)$, is a low-rank matrix, and thus not invertible. To overcome this limitation, we propose to remove the variable, $\mathbf{v}_{t}$, from the ELB, by rather using pdfs that are conditional on this variable (i.e., for which $\mathbf{v}_{t}$ is a fixed known value). Since the iterations' process occurs in the second update step (i.e., which uses $\mathbf{z}_{t}$ ), we assign to $\mathbf{v}_{t}$ the mean $\hat{\boldsymbol{\eta}}_{t}$ of $\left\{\boldsymbol{\eta}_{t}^{(i)}\right\}_{i=1}^{m}$, which, indeed, is an approximation of $\mathbb{E}_{q\left(\boldsymbol{\xi}_{t} \mid \mathbf{r}_{0: t-1}, \mathbf{y}_{t}\right)}\left[\mathbf{v}_{t}\right]$ (i.e., the unconstrained analysis mean of $\mathbf{v}_{t}$ ). The resulting ELB reads,

$$
\begin{aligned}
\mathcal{E}_{2} & =\mathbb{E}_{q\left(\boldsymbol{\Sigma} \mid \mathbf{r}_{0: t}\right)}\left[\ln \left(p\left(\boldsymbol{\Sigma}, \mathbf{r}_{t} \mid \mathbf{r}_{0: t-1}, \hat{\boldsymbol{\eta}}_{t}\right)\right)\right]-\mathbb{E}_{q\left(\boldsymbol{\Sigma} \mid \mathbf{r}_{0: t}\right)}\left[\ln \left(q\left(\boldsymbol{\Sigma} \mid \mathbf{r}_{0: t}\right)\right)\right] \\
& \approx \operatorname{cte}+\mathbb{E}_{q\left(\boldsymbol{\Sigma} \mid \mathbf{r}_{0: t}\right)}\left[\ln \left(p\left(\mathbf{z}_{t} \mid \boldsymbol{\Sigma}, \hat{\boldsymbol{\eta}}_{t}\right)\right)\right]+\mathbb{E}_{q\left(\boldsymbol{\Sigma} \mid \mathbf{r}_{0: t}\right)}\left[\ln \left(q\left(\boldsymbol{\Sigma} \mid \mathbf{r}_{0: t-1}\right)\right)\right]-\mathbb{E}_{q\left(\boldsymbol{\Sigma} \mid \mathbf{r}_{0: t}\right)}\left[\ln \left(q\left(\boldsymbol{\Sigma} \mid \mathbf{r}_{0: t}\right)\right)\right]
\end{aligned}
$$


where the term "cte" encompasses all the terms that do not depend on $\Sigma$. This suggests that the convergence of the proposed scheme can be monitored based either on the change in $\mathcal{E}_{2}$ only, the change in RSEN of the state only, the change in $\mathcal{E}_{2}$ and RSEN of the state, or, as stated above, the change in RSEN of both state and $\boldsymbol{\Sigma}$. Finally, based on the Gaussian expression of $p\left(\mathbf{z}_{t} \mid \boldsymbol{\Sigma}, \hat{\boldsymbol{\eta}}_{t}\right)$ and the inverse-Gamma expression of $q\left(\boldsymbol{\Sigma} \mid \mathbf{r}_{0: t-1}\right)$ and $q\left(\boldsymbol{\Sigma} \mid \mathbf{r}_{0: t}\right)$, one readily shows that Eq. (21) at iteration $(\ell) \rightarrow(\ell+1)$ is given as,

$$
\mathcal{E}_{2}^{(\ell)} \approx \operatorname{cte}+\frac{\hat{\alpha}_{t}}{\hat{\beta}_{t}^{(\ell+1)}}\left[\hat{\beta}_{t}^{(\ell+1)}-\hat{\beta}_{t-1}-\left\|\mathbf{z}_{t}-\mathbf{M} \hat{\boldsymbol{\eta}}_{t}\right\|^{2} / 2\right]-\ln \left(\hat{\beta}_{t}^{(\ell+1)}\right),
$$

where cte gathers the terms that do not vary with iterations (i.e., independent of $(\ell)$ ).

The adaptation of the algorithm above to the case of an inhomogeneous noise with a covariance is straightforward,

$$
\boldsymbol{\Sigma}=\operatorname{diag}\left(\lambda^{1}, \cdots, \lambda^{n_{z}}\right)
$$

where $\operatorname{diag}(\mathbf{v})$ denotes a diagonal matrix with diagonal v. More specifically, Eqs. (11)-(16) that compute the state ensembles are kept unchanged, and only those related to the noise variance will be updated (i.e., Eqs. (17)-(19) for each $\lambda^{j}$ ). Each variance $\lambda^{j}, \quad j=1, \cdots, n_{z}$, is estimated separately from the others, $\lambda^{k}, \quad k \neq j$, by a direct application of Eqs. (17)-(19) and (22), which, correspond to the $n_{z} \times 1$ vectorial model (3), on the scalar (marginal) model,

$$
\mathbf{z}_{t, j}=\mathbf{G}(j,:) \mathbf{x}_{t}+\mathbf{L}(j,:) \mathbf{x}_{t-1}+\boldsymbol{\xi}_{t, j}
$$

where $\mathbf{z}_{t, j}$ and $\boldsymbol{\xi}_{t, j}$ respectively denote the $j^{t h}$ component of $\mathbf{z}_{t}$ and $\boldsymbol{\xi}_{t}$ (i.e., $\boldsymbol{\xi}_{t, j} \sim \mathcal{N}\left(\mathbf{0}, \lambda^{j}\right)$ ), and $\mathbf{G}(j,:)$ and $\mathbf{L}(j,:)$ are the $j^{\text {th }}$ rows of $\mathbf{G}$ and $\mathbf{L}$, respectively. A schematic illustration of this algorithm is presented in Figure 2 .

FIGURE 2 


\section{Experimental setup}

\subsection{Data merging}

A single product for each water flux term of precipitation $(\mathbf{p})$ and evaporation $(\mathbf{e})$ is required to close the water balance in the second update step of UWCEnKF. One can use only one data product for each flux components, e.g., only TRMM-3B43 for $\mathbf{p}$ for the filtering process. However, this may introduce errors because various products are subject to a different rate of uncertainty over different areas. Alternatively, the different data products for each component can be merged into a unique $\mathbf{p}$ and $\mathbf{e}$ to better represent the water balance over the globally distributed basins (Sahoo et al., 2011). Here, we merge various datasets of precipitation and evaporation prior to data assimilation. To this end, we follow Sahoo et al. (2011) and merge the data considering their relative error levels w.r.t. non-satellite products. This combination is done in a way that satellitebased products are merged to be used in data assimilation while other products are only applied for the merging objective. For $\mathbf{p}$, the average of GPCC and CPC unified gauge over each basin is assumed as the truth and is used to estimate the error level of each satellite-based product, i.e, TRMM-3B43, CMORPH, and GPCP. A similar strategy is applied for evaporation, where ERA-interim and VIC products are used to quantify the error level associated with the data of MOD16 and GLEAM outputs that are based on satellite products (Miralles et al., 2011). It is worth mentioning that a more robust merging process can be achieved by involving ground-based measurements as a reference rather than ERA-interim and VIC. Obtaining and analyzing such an enhanced evaporation dataset from in-situ stations over all tested basins is however very difficult and is out of the scope of this study. Therefore, we use these model outputs to merge satellite-based datasets into a single e. Once the references are calculated, we use a multiplicative error model to estimate the offset, scale parameter, and error variance for each data product. These variances are then used to compute the observations weights as,

$$
w_{i}=\frac{1}{\sigma_{i}^{2}} / \sum_{k=1}^{n_{p}} \frac{1}{\sigma_{k}^{2}} .
$$

For each data product $(i)$, using the error variances of that specific product $\sigma_{i}^{2}$ and all products $\left(\sigma_{k}^{2}\right)$ in the same data type (with the total number of $n_{p}$ ), weight $w_{i}$ can be calculated. Eq. (25) is applied for both precipitation and evaporation to provide merged data with reduced error (Luo et al., 2007; Sahoo et al., 2011). Note that the above approach is applied only to merge the various 
data products and to obtain uniform precipitation and evaporation datasets prior to assimilation. The estimated errors (e.g., $\sigma_{i}^{2}$ in Eq. (25)) are used only for this objective and are not related to the water flux error covariance calculation in the filtering procedure (cf. Section 3.2).

\subsection{Data assimilation}

To start the assimilation process, the initial ensemble is generated by perturbing the forcing fields. To this end, we use Monte Carlo sampling to perturb the precipitation, shortwave radiation, and temperature field considering a Gaussian multiplicative error of $30 \%$ for precipitation, an additive Gaussian error of $50 \mathrm{Wm}^{-2}$ for the shortwave radiation, and a Gaussian additive error of $2^{\circ} \mathrm{C}$ for temperature (Jones et al., 2007). The system state includes top soil, shallow soil, deep soil water, snow, vegetation, surface, and groundwater storages. Except for groundwater and surface storage, all the other components are simulated with two hydrological response units (HRU) of tall, e.g., deep-rooted vegetation and short, e.g., shallow-rooted vegetation. This leads to a state vector of dimension $(2 \times 5+1+1) \times 1695$ (corresponding to 1695 grid points over all basins).

All observations, including GRACE TWS, satellite soil moisture data, and water fluxes are assimilated monthly. The monthly increment is then be added to each day of the current month, which guarantees that the update of the monthly mean is identical to the monthly mean of the daily updates. Here, the differences between the predictions and the updated state variables are added as offsets to the state variables at the last day of each month to generate the ensembles for the next month assimilation step (see Eicker et al., 2014, for more details). The observation operator aggregates different water storages at each grid point to update with GRACE TWS and scales the top-layer soil storage by the field capacity value to provide a relative wetness for updating with the soil moisture products of AMSR-E and SMOS (Renzullo et al., 2014).

In addition, observation error covariances for the first update step are required. Full error information about the Stokes' coefficients are used to construct the TWS error covariance matrix. This is done by converting GRACE spherical harmonic error coefficients to TWS error covariances following Khaki et al. (2017c). Since such an information is not available for soil moisture products, we assume their error covariances to be uncorrelated with standard deviations of $0.04 \mathrm{~m}^{3} \mathrm{~m}^{-3}$ for SMOS (as suggested by Leroux et al., 2016) and $0.05 \mathrm{~m}^{3} \mathrm{~m}^{-3}$ for AMSR-E (as suggested by De Jeu et al., 2008). We further apply two common auxiliary techniques of ensemble variance inflation and covariance localization to mitigate for the ensemble spread collapse and rank deficiency (Anderson 
et al., 2001; Houtekamer and Mitchell, 2001). These include an ensemble inflation with a coefficient factor of 1.12 and Local Analysis (LA) with a localization length scale of $5^{\circ}$ (see Khaki et al., 2017b, for more details).

\section{Results}

The results are discussed in three parts. UWCEnKF implementation is first presented and discussed in Section 5.1.1. The validation of the proposed approach against in-situ groundwater and soil moisture measurements is then presented in Section 5.2. The relevance of the second update step in UWCEnKF and its overall effects on the assimilation system performance is finally analyzed in Section 5.3. UWCEnKF estimates are also compared with the results of WCEnKF and EnKF. UWCEnKF is tested with both constant (Structure in Eq. (10), indicated by UWCEnKF-1) and spatially varying (Structure in Eq. (23), indicated by UWCEnKF-2) error variances for the water balance equation. While UWCEnKF-1 assigns a fixed error variance to water fluxes at all points, different values for individual points are calculated by UWCEnKF-2.

\subsection{Implementation results}

\subsubsection{Iteration impacts}

We first study the sensitivity of UWCEnKF-1, and UWCEnKF-2 to the iteration procedure. As mentioned, in contrast with WCEnKF, which assumes that these uncertainties are known, UWCEnKF estimates the error covariance through an iteration process. To show how this iteration works, we compare the convergence of UWCEnKF-1 and UWCEnKF-2, based on Eq. (22), in Figure 3. The average evolutions of $\mathcal{E}_{2}^{(\ell+1)}-\mathcal{E}_{2}^{(\ell)}$ (the difference between Eq. (22) in each two successive iterations) from both filters for $\ell=0 \cdots 10$ are shown in this figure. After few iterations, generally less than 8, both UWCEnKF-1 and UWCEnKF-2 converge. Faster convergence and lower differences $\mathcal{E}_{2}^{(\ell+1)}-\mathcal{E}_{2}^{(\ell)}$ are also generally achieved by UWCEnKF-2 compared to UWCEnKF-1. It can be seen that after 5 iterations, UWCEnKF-2 decreases to a value below the selected arbitrary threshold of $\mathcal{E}_{2}^{(\ell+1)}-\mathcal{E}_{2}^{(\ell)}=10 \mathrm{~mm}$. This is due to the fact that UWCEnKF-2 enables more degree of freedom in the optimization process by using different error variance for each grid point as compared to UWCEnKF-1, which tries to fit a single value for the entire domain.

\section{FIGURE 3}


In order to demonstrate the relevance of the UWCEnKF, we compare its results against those of the WCEnKF wit hvarious preselected values of error variances. The sensitivity of the WCEnKF to the choice of $\boldsymbol{\Sigma}$ can be seen in Figures 4. The various implementations of the WCEnKF result in different performances in terms of imbalance and the Root-Mean-Squared Error (RMSE), which is calculated based on the assimilation results and groundwater in-situ measurements over the Murray-Darling Basin. The estimated groundwater time series from the WCEnKF and UWCEnKF are spatially interpolated to the nearest gauge stations. The difference between in-situ and filtered time series are then used to calculate the RMSE.

\section{FIGURE 4}

Each circle in Figures 4 refers to the average results of an independent implementation of WCEnKF. It can be seen that the results of this filter largely vary depending on the selection of the error variance. Overall, lower imbalance and RMSE are obtained by assuming 20 to $30 \mathrm{~mm}^{2}$. UWCEnKF-1 and UWCEnKF-2, on the other hand, achieve better results, shown by the triangle and cross, respectively, in a single implementation. The optimization algorithms used in UWCEnKF cause this independence of the error variance choice. It can also be seen that WCEnKF can achieve comparable results to that of UWCEnKF-1 in few cases. UWCEnKF-2, however, generally leads to the minimum RMSE and imbalance.

\subsubsection{Spatial and temporal balance error variance}

The performance of the proposed UWCEnKF in estimating water balance error variance and their effects on the imbalance between water fluxes are discussed in this section and is further compared with WCEnKF results. Both spatial and temporal variabilities are examined. Figure 5 shows the temporally averaged error variances assigned to the observations for WCEnKF, as well as those estimated by UWCEnKF-1 and UWCEnKF-2 over the Amazon Basin. It can be seen that UWCEnKF-1 and UWCEnKF-2 estimate different errors at each iteration. The error variance maps in WCEnKF, on the other hand, is fixed to what has been assigned prior to data assimilation. After eight iterations, it is observed that the error estimated by UWCEnKF-1 is closer to the average of UWCEnKF-2 results $\left(34.70 \mathrm{~mm}^{2}\right)$, i.e., $41.19 \mathrm{~mm}^{2}$ for UWCEnKF-1 and, in comparison to $68.74 \mathrm{~mm}^{2}$ for WCEnKF. This indicates that both UWCEnKF-1 and UWCEnKF2 result in uncertainties with close magnitude for water balances and the implemented algorithms 
allow for such an adjustment during iteration steps. Furthermore, Figure 5 depicts the spatial variability characteristics of error variances estimated by UWCEnKF-2. This property allows for more flexibility for error adjustment in UWCEnKF-2. These flexibilities in the UWCEnKF filtering method, as illustrated in Figure 6, result in a smaller imbalance.

\section{FIGURE 5}

\section{FIGURE 6}

The better performances of UWCEnKF-1 and UWCEnKF-2 compared to WCEnKF in minimizing imbalance errors are clear in Figure 6, where each map shows the estimated imbalance corresponding to Figure 5 setups. Figure 6 shows that the iteration algorithm effectively reduces imbalance errors, even after only few iterations (e.g., four). In addition, it can be seen that the applied algorithm in UWCEnKF provides the opportunity for error variances to be adjusted with no supervision as in WCEnKF. UWCEnKF-2, with more flexibility for such adjustment than UWCEnKF-1 (cf. Figure 5), leads to the smaller imbalance, that is $\sim 6 \mathrm{~mm}$ (absolute average of all values) against $\sim 13 \mathrm{~mm}$ (on average) for UWCEnKF-1. This larger improvement for UWCEnKF-2 results is achieved by estimating different error variance values over each grid point, and correspondingly applying different rate of adjustments (based on the estimated water balance uncertainty) from the equality constraint to the points.

An example of the abovementioned spatially varying error variance in UWCEnKF-2 can be seen in Figure 7. Figure 7a depicts the average imbalance over Murray-Darling basin after jointly assimilating GRACE TWS and satellite soil moisture in the first analysis step of UWCEnKF. It is worth mentioning that we find larger impacts of GRACE TWS data (approximately 7.5 times for all the basins) on the imbalance between fluxes compared to the satellite soil moisture products, which could be explained by the fact that contrary to the soil moisture assimilation, GRACE data influences all compartments. The temporally averaged estimated variances are displayed in Figure 7b. It can be seen that both estimated maps exhibit similar spatial patterns in some areas. One can also see in Figure 7b that, in general, a larger variance is estimated over the areas with larger imbalance. Figure 7c shows the average applied increments in the second analysis step of UWCEnKF-2 to account for the above imbalances. It is clear that larger increments are applied over the areas with larger imbalances, e.g., the north, southeast, and southwest parts of the basin. 
The areas such as the central parts, which display smaller imbalance in Figure 7a, are also assigned smaller increments as shown in Figure 7c.

\section{FIGURE 7}

Similar flexibilities for error variance estimation in UWCEnKF can also be seen from the temporal variabilities of error variances as demonstrated in Figure 8. The water balance error variances at each assimilation step are estimated from UWCEnKF-1 for the entire Orange Basin and from UWCEnKF-2 for each grid point (green shaded area) of the basin. The figure also plots that of UWCEnKF-2 derived spatially averaged values, as well as errors used in WCEnKF. Again, it is clear from Figure 8 that UWCEnKF-1 and UWCEnKF-2 allow for larger variations in error estimations than WCEnKF. It can also be seen that errors at each point can vary independently in UWCEnKF-2, which results in a better uncertainty adjustment. This can help for optimal imbalance minimization in the filter.

FIGURE 8

FIGURE 9

UN

42

3

(ct.

475

476

477

Both spatial and temporal variabilities of error variances are summarized in Figure 9 over all basins, which shows variation ranges of water balance covariance in time (vertical lines) and space (horizontal lines) for WCEnKF, UWCEnKF-1, and UWCEnKF-2. In contrast to WCEnKF and UWCEnKF-1, spatial variabilities can be observed in UWCEnKF-2 results. As discussed, this helps for a better error adjustment during the filtering process. In terms of temporal variations, both UWCEnKF-1 and UWCEnKF-2 perform comparably well representing a larger range of changes than WCEnKF over all basins. The unsupervised error estimation algorithm in UWCEnKF enables to estimate an "optimal" water balance error calculation, which as it will be shown in Section 5.3 (cf. Figure 15) leads to smaller imbalance errors. In cases where assigned error to WCEnKF is close to what is calculated by UWCEnKF, e.g., Indus Basin, the final achieved imbalance from the filters are also close. In other cases with larger differences between assigned and estimated errors, there are larger discrepancies in imbalances. 


\subsection{Validations with in-situ measurements}

The performances of the EnKF and UWCEnKF are compared with in-situ measurements. UWCEnKF was tested with both constant (UWCEnKF-1) and spatially varying (UWCEnKF-2) error variances for the water balance equation. Figure 10 shows the average groundwater time series over the Mississippi, Murray-Darling and the St. Lawrence basins, estimated by the openloop run (without assimilation), EnKF, WCEnKF, UWCEnKF-1, and UWCEnKF-2. Remarkable improvement can be seen from the different filters compared to the open-loop time series. In this regard, WCEnKF and UWCEnKF generally perform better than EnKF. This is more evident when a considerable trend exists in the time series, e.g., within the Murray-Darling basin after 2009 and St. Lawrence between 2010 and 2012. It can also be seen that UWCEnKF groundwater time series in most of the times better match to those of in-situs. A clear example of this can be found in Murray-Darling basin 2011-2013. Furthermore, comparing UWCEnKF-1 and UWCEnKF-2, better agreements between in-situ and estimated groundwater changes are achieved for UWCEnKF-2 over all three basins, particularly in the Mississippi basin.

FIGURE 10

To better monitor how UWCEnKF improves the groundwater estimates, their results are compared with in-situ measurements and against those of EnKF. RMSE and standard deviation (STD) are calculated for groundwater error time series, i.e., the difference between in-situ and filtered groundwater time series, at the location of each in-situ station. Figures 11 and 12 display the results over the Murray-Darling and Mississippi basins, respectively.

FIGURE 11

FIGURE 12

One can see that the filters successfully reduce RMSE and STD w.r.t. the open-loop run. This indicates the relevance of assimilation for decreasing state estimate errors. The groundwater estimate improvements are different for each filter. UWCEnKF-1 and UWCEnKF-2 suggest more (18\% on average) error reduction than EnKF. Overall, more pronounced error reductions are achieved over the Mississippi basins, which could be attributed to larger model errors within the 
basin. Slightly better performances $(\sim 4 \%)$ in terms of groundwater error reduction are obtained with UWCEnKF-2 compared to UWCEnKF-1. We also compute the correlations (at 0.05 significance level) between the filtered and in-situ groundwater time series. Similarly, larger correlations result from the filter estimates compared to the open-loop run, namely, $14 \%$ from EnKF, $26 \%$ for UWCEnKF-1, and $29 \%$ for UWCEnKF-2. The correlation results also confirm that UWCEnKF provides better estimates of the groundwater time series.

In-situ soil moisture measurements are also used to assess the assimilation impact on soil storage. To this end, similar to groundwater assessment, filtered soil moisture time series at the stations' locations are compared with their in-situ counterpoints at different layers. Figure 13 shows root-zone soil moisture variation time series as estimated by the various filters, as well as in-situ measurements over the Mississippi, Murray-Darling, St. Lawrence, Danube, and the Yangtze basins. It can be seen that all filters decrease the misfits between estimated and measured soil moisture variations. In some cases, however, UWCEnKF, and to a lesser degree WCEnKF, performs better, e.g., Mississippi (2009), Murray-Darling (2004 and 2008), and Danube (2006). There are also various occasions during which the WCEnKF and UWCEnKF-1 results are very close, such as St. Lawrence 2010-2012 and Yangtze 2005-2006. This can be explained by the fact that both methods use a single error variance value for water balance uncertainties, so whenever a good approximation is used to assign this value prior to data assimilation in WCEnKF, close to what is estimated in UWCEnKF-1, the corresponding state estimates seen to be also close. UWCEnKF-2, on the other hand, performs relatively better, being more successful in matching soil moisture estimates to the in-situ soil moisture variations.

FIGURE 13

The correlation results between the monthly soil moisture estimates for all filters w.r.t. the monthly in-situ measurements are presented in Table 2. Note that different soil moisture estimates of various soil layers are compared to soil moisture measurements at corresponding layers and their average are reported in the table. For instance, the model top layer is compared with $0-8$ $\mathrm{cm}$ measurements over the Murray-Darling basin and 0-10 $\mathrm{cm}$ over Mississippi basin, summations of the model top, shallow, and a small portion of deep-root soil layers are tested against 0-30 $\mathrm{cm}$ and 0-50 $\mathrm{cm}$ measurements over the Murray-Darling and Mississippi basins, respectively, and 
summations of the model's soil layers are compared to 0-90 cm (for Murray-Darling) and 0-100 $\mathrm{cm}$ (for Mississippi) soil measurements. Due to a difference between the soil moisture estimates (i.e., column water storage measured in $\mathrm{mm}$ ) and the in-situ measurements (i.e., volumetric soil moisture), only a correlation analysis is conducted. Additionally, in order to statistically assess the results, a significance test for the correlation coefficients is applied based on the t-distribution. The estimated t-value and the distribution at 0.05 significant level are used to calculate the p-value, which is assumed to be significant if it lies under $5 \%$.

\section{TABLE 2}

The results indicate that assimilation significantly improves soil storages regardless of the applied filter. All the filters have positive effects on soil moisture estimates. UWCEnKF performs better than both WCEnKF and EnKF with respectively $6 \%$ and $11 \%$ higher correlations with the in-situ measurements. It can also be seen that in some cases, e.g., Mississippi basin, the filters generally perform comparably, especially WCEnKF and UWCEnKF-1. This indicates that WCEnKF is capable of improving soil moisture estimates as UWCEnKF subject to using an accurate water balance uncertainty because this is the only difference between the two approaches. The largest improvement with an average $20.28 \%$ for all basins is achieved by UWCEnKF-2, better than UWCEnKF-1 (17.75\% on average) and noticeably larger than EnKF (7.85\%).

We further examine the assimilation results against independent discharge data over different basins. It is worth mentioning that these discharge datasets are not assimilated. The average correlations between the estimated water discharge time series and those from the in-situ data over each basin are presented in Table 3. Improvements are achieved for all assimilation experiments w.r.t. the open-loop run. The EnKF increases the correlation by $4 \%$ (on average), while UWCEnKF-1 and UWCEnKF-2 increase the correlation by approximately $23 \%$ and $24 \%$, respectively. Again, UWCEnKF provides better results than EnKF over all basins. The largest correlation values are obtained for the Murray-Darling and Amazon basins, while the largest correlation improvements are achieved over the Orange, Amazon, and the Yangtze basins.

TABLE 3 


\subsection{Impact of the equality constraint}

To further investigate the relevance of the second analysis step of UWCEnKF, we calculate correlations between the filters estimates and assimilated observations at the forecast and analysis steps for all basins. The average correlations improvements w.r.t. the open-loop run are plotted in Figure 14. As expected, larger correlations are obtained in the analysis step. In general, applying EnKF results in larger correlations between the estimates and assimilated observations (e.g., GRACE TWS and AMSR-E+SMOS) because during the EnKF assimilation the full magnitude of the update is applied to the variables regardless of the water balance. However, the WCEnKF and UWCEnKF take into account the water balance in a second update, which leads to the most improvements regarding $\mathbf{p}, \mathbf{e}$, and $\mathbf{q}$. This is due to the fact that the first update in the WCEnKF and UWCEnKF corrects the state variables with the observations, and the second update corrects the water balance. This suggests that water budget constraint slightly degrades the effects of observations in the (second) update step in both WCEnKF and UWCEnKF filters, which is generally due to the observation overfitting problem, when no constraint is applied (e.g., standard EnKF) in data assimilation (see also Tangdamrongsub et al., 2017; Khaki et al., 2017a). Furthermore, there is a degree of disagreement between TWS changes and other flux observations (e.g., precipitation, evaporation, and discharge), which could be attributed to different sources of uncertainties in the observations (see, e.g., Aires, 2014; Munier et al., 2015). The water budget constraint applied to data assimilation (i.e., the second update of UWCEnKF) accounts for this effect by further correcting the estimated states from the first update step based on GRACE TWS. The second step partly removes the artifacts from data assimilation of GRACE in the first step. It can clearly be seen that UWCEnKF provides higher correlations to the flux observations than WCEnKF. This improvement is more pronounced by using UWCEnKF-2. UWCEnKF's both variants remarkably increase the correlations between TWS estimates and water fluxes compared to EnKF. Overall, a better performance is observed for UWCEnKF-2 in comparison to UWCEnKF-1.

FIGURE 14

The results of water budget closure resulting from each filter for every basin are shown in Figure 15. UWCEnKF-1 and UWCEnKF-2 clearly reduce water budget imbalances for all basins compared to WCEnKF and especially EnKF. It can also be seen that UWCEnKF-2 better enforces the balance 
between water components after assimilation. The absolute imbalance from UWCEnKF-2 is 15.28 mm, 8.26\% smaller than UWCEnKF-1, 17.84\% smaller than WCEnKF, and $36.47 \%$ smaller than EnKF. Note that these average values are computed for all basins. The imbalance reductions can also be seen from the reported STD values for each time series in Figure 15. In all basins, the largest STD results from the EnKF and the least from the UWCEnKF-2. In some cases such as Indus, and to a lesser degree Amazon, WCEnKF performs comparably to UWCEnKF-1. UWCEnKF-2, on the other hand, achieves the largest water budget imbalance reduction, in terms of amplitude and STD, which confirms the results of Figure 14, as well as the validation results against in-situ measurements.

FIGURE 15

\section{Conclusions}

This study introduced an Unsupervised Weak Constrained Ensemble Kalman Filter (UWCEnKF) to mitigate for water budget imbalance while accounting for uncertainties in the inputs of the water balance components. UWCEnKF is an extension of the previously proposed Weak Constrained Ensemble Kalman Filter (WCEnKF) to a more general (unsupervised) framework, in which the covariance associated with the water balance model is estimated along with the system state. Numerical experiments were carried out to assess the performance of the UWCEnKF against WCEnKF, as well as the standard Ensemble Kalman Filter (EnKF). The filters' results examinations against available in-situ measurements indicated that UWCEnKF performs best in terms of groundwater error reduction and soil moisture estimate improvements. In general, UWCEnKF reduced groundwater errors (w.r.t. groundwater in-situ measurements) by $18 \%$ (on average), and $11 \%$ (on average) more than EnKF and WCEnKF, respectively. UWCEnKF-2 also achieved $4 \%$ (on average) smaller groundwater RMSE than UWCEnKF-1. Furthermore, UWCEnKF increased the correlation values between soil moisture estimates and those of the in-situ measurements by $6 \%$ more than WCEnKF and 12\% more than EnKF. Again, UWCEnKF-2 performed better than UWCEnKF-1 with larger soil moisture correlations w.r.t. the in-situ soil moisture measurements, i.e., $20.28 \%$ against $17.75 \%$. UWCEnKF also achieved larger correlations to independent discharge datasets, e.g., respectively $6 \%$ and $11 \%$ larger correlations with the in-situ measurements than WCEnKF and EnKF. The experiments results also suggested that the UWCEnKF using spatially 
varying error variances for the water balance equation provides better groundwater and soil moisture estimates than applying a constant error variance. A similar performance was also obtained for the water budget imbalance reduction, where the prior variant better mitigated the imbalance problem than the latter case.

Overall, UWCEnKF achieved maximum correlations with the flux observations, both during the forecast and analysis steps. The largest imbalance reduction was also obtained using UWCEnKF. More specifically, the absolute imbalance for UWCEnKF-2 is $15.28 \mathrm{~mm}, 8.26 \%$ smaller than UWCEnKF-1, 17.84\% smaller than WCEnKF, and 36.47\% smaller than EnKF. These results demonstrate the relevance of the new proposed unsupervised scheme, which is straightforward to implement and computationally not intensive. Future work will consider extending the proposed framework to jointly estimate the model biases with the state and the observation error variance.

\section{Acknowledgement}

\section{References}

Adler, R.F., Susskind, J., Huffman, G.J., Bolvin, D., Nelkin, E., Chang, A., et al., (2003). Global precipitation climatology project V2.1 monthly 2.5 deg global 1979present (satellite only and gauge adjusted) 2003: The version-2 global precipitation climatology project (GPCP) monthly precipitation analysis (1979present). Journal of Hydrometeorology, 4, 11471167.

Ait-El-Fquih, B., Hoteit, I., (2015). Fast Kalman-like Filtering in large-dimensional linear and Gaussian state-space models. IEEE Transactions on Signal Processing, 63, 5853-5867.

Ait-El-Fquih, B., Hoteit, I., (2016). A variational Bayesian multiple particle filtering scheme for large-dimensional systems. IEEE Transactions on Signal Processing, 64, 5409-5422.

Anderson, J., (2001). An Ensemble Adjustment Kalman Filter for Data Assimilation. Mon. Wea. Rev., 129, 28842903, http://dx.doi.org/10.1175/1520-0493(2001)129¡2884:AEAKFF ¿2.0.CO;2. 
Aires, F., (2014). Combining datasets of satellite retrieved products. Part I: Methodology and water budget closure. Journal of Hydrometeorology, 15 (4), 16771691.

Blei, David M., Alp Kucukelbir, and Jon D. McAuliffe (2017). Variational Inference: A Review for Statisticians. Journal of the American Statistical Association, 112:518, 859-877, DOI: $10.1080 / 01621459.2017 .1285773$.

Chen, M., Xie, P., Janowiak, J.E., Arkin, P.A., (2002). Global land precipitation: A 50-yr monthly analysis based on gauge observations. Journal of Hydrometeorology, 3, 249266.

Cheng, M.K., Tapley, B.D., (2004). Variations in the Earth's oblateness during the past 28 years. Journal of Geophysical Research, Solid Earth, 109, B09402. http://dx.doi.org/10.1029/2004JB003028.

De Jeu, R.A.M., Owe, M., (2003). Further validation of a new methodology for surface moisture and vegetation optical depth retrieval. Int J Remote Sens 24:45594578, http://dx.doi.org/10.1080/0143116031000095934.

De Jeu, R.A.M., Wagner, W., Holmes, T.R.H., Dolman, A.J., van de Giesen , N.C., Friesen J., (2008) Global Soil Moisture Patterns Observed by Space Borne Microwave Radiometers and Scatterometers, Surveys in Geophysics,Volume 29, Issue 45, pp 399420, http://dx.doi.org/10.1007/s10712-008-9044-0.

Doll, P., Kaspar, F., Lehner, B., (2003). A global hydrological model for deriving water availability indicators: model tuning and validation, J. Hydrol., 270, 105134.

Draper, C.S., Mahfouf, J.-F., Walker, J.P., (2009), An EKF assimilation of AMSRE soil moisture into the ISBA land surface scheme, J. Geophys. Res., 114, D20104, http://dx.doi.org/10.1029/2008JD011650.

Drusch, M., Wood, E.F., Gao, H., (2005). Observation operators for the direct assimilation of TRMM microwave imager retrieved soil moisture. Geophysical Research Letters, 32, L15403.

Eicker, A., Schumacher, M., Kusche, J., Dll, P., Mller-Schmied, H., (2014). Calibration/data assimilation approach for integrating GRACE data into the WaterGAP global hydrology model (WGHM) using an ensemble Kalman filter: first results, SurvGeophys, 35(6):12851309. http://dx.doi.org/10.1007/s10712-014-9309-8. 
Gutentag, E.D., Heimes, F.J., Krothe, N.C., Luckey, R.R., Weeks, J.B., (1984). Geohydrology of the High Plains aquifer in parts of Colorado, Kansas, Nebraska, New Mexico, Oklahoma, South Dakota, Texas, and Wyoming, U.S. Geol. Surv. Prof. Pap., 1400-B, 66 pp.

Han, R., Tian, X.J., Fu, Y., Cai, Z.N., (2015). Real-data assimilation experiment with a joint data assimilation system: assimilating carbon dioxide mole fraction measurements from the Greenhouse gases Observing Satellite, Atmospheric and Oceanic Science Letters, Volume 9, Issue 2, Pages 107-113, https://doi.org/10.1080/16742834.2016.1133070.

Houtekamer, P.L., Mitchell, H.L., (2001). A Sequential Ensemble Kalman Filter for Atmospheric Data Assimilation, Mon. Wea. Rev., 129:1, 123-137.

Huffman, G.J., Adler, R.F., Bolvin, D.T., Gu, G., Nelkin, E.J., Bowman, K.P., Hong, Y., Stocker, E.F., Wolff, D.B., (2007). The TRMM Multi-satellite Precipitation Analysis: Quasi- Global, Multi-Year, Combined-Sensor Precipitation Estimates at Fine Scale. J. Hydrometeor., 8(1), 3855.

Jaakkola, T.S., Jordan, M.I., (2000). Bayesian parameter estimation via variational methods. Statistics and Computing, 10, 25-37.

Jackson, T., Bindlish, R., (2012). Validation of Soil Moisture And Ocean Salinity (SMOS) soil moisture over watershed networks in the US, IEEE Trans. Geosci. Remote Sens., 50, 15301543.

Jones, D.A., Wang, W., Fawcett, R., Grant, I., (2007). Climate data for the Australian water availability project. In: Australian Water Availability Project Milestone Report. Bur. Met., Australia, 37pp.

Joyce, R.J., Janowiak, J.E., Arkin, P.A., Xie, P.P., (2004). CMORPH: A method that produces global precipitation estimates from passive microwave and infrared data at high spatial and temporal resolution. Journal of Hydrometeorology, 5, 487503.

Khaki, M., Ait-El-Fquih, B., Hoteit, I., Forootan, E., Awange, J., Kuhn, M., (2017a). A Two-update Ensemble Kalman Filter for Land Hydrological Data Assimilation with an Uncertain Constraint, Journal of Hydrology, Volume 555, Pages 447-462, ISSN 0022-1694, https://doi.org/10.1016/j.jhydrol.2017.10.032. 
Khaki, M., Schumacher, M., J., Forootan, Kuhn, M., Awange, E., van Dijk, A.I.J.M., (2017b). Accounting for Spatial Correlation Errors in the Assimilation of GRACE into Hydrological Models through localization, Advances in Water Resources, Volume 108, Pages 99-112, ISSN 0309-1708, https://doi.org/10.1016/j.advwatres.2017.07.024.

Khaki, M., Hoteit, I., Kuhn, M., Awange, J., Forootan, E., van Dijk, A.I.J.M., Schumacher, M., Pattiaratchi, C., (2017c). Assessing sequential data assimilation techniques for integrating GRACE data into a hydrological model, Advances in Water Resources, Volume 107, Pages 301-316, ISSN 0309-1708, http://dx.doi.org/10.1016/j.advwatres.2017.07.001.

Khaki, M., Forootan, E., Kuhn, M., Awange, J., Papa, F., Shum, C.K., (2018a). A Study of Bangladesh's Sub-surface Water Storages Using Satellite Products and Data Assimilation Scheme. Science of The Total Environment, 625:963-977, https://doi.org/10.1016/j.scitotenv.2017.12.289.

Khaki, M., Forootan, E., Kuhn, M., Awange, J., van Dijk, A.I.J.M., Schumacher, M., Sharifi, M.A., (2018b). Determining Water Storage Depletion within Iran by Assimilating GRACE data into the W3RA Hydrological Model. Advances in Water Resources, 114:1-18, https://doi.org/10.1016/j.advwatres.2018.02.008.

Khaki, M., Forootan, E., Kuhn, M., Awange, J., Longuevergne, L., Wada, W., (2018c). Efficient Basin Scale Filtering of GRACE Satellite Products, Remote Sensing of Environment, Volume 204, Pages 76-93, ISSN 0034-4257, https://doi.org/10.1016/j.rse.2017.10.040.

Kusche, J., Schmidt R., Petrovic, S., Rietbroek, R., (2009). Decorrelated GRACE time-variable gravity solutions by GFZ and their validation using a hydrological model, Journal of Geodesy, http://dx.doi.org/10.1007/s00190-009-0308-3.

Leroux, D.J., Pellarin, T., Vischel, T., Cohard, J.-M., Gascon, T., Gibon, F., Mialon, A., Galle, S., Peugeot, C., Seguis, L., (2016). Assimilation of SMOS soil moisture into a distributed hydrological model and impacts on the water cycle variables over the Oum catchment in Benin, Hydrol. Earth Syst. Sci., 20, 2827-2840, https://doi.org/10.5194/hess-20-2827-2016.

Liang, X., Lettenmiar, D.P.,Wood, E.F., Burges, S.J., (1994). A simple hydrologically based model of landsurfacewater and energy fluxes for general-circulationmodels. Journal of Geophysical Research, 99, 14,41514,428. http://dx.doi.org/10.1029/94JD00483. 
Lievens, H., Reichle, R.H., Liu, Q., De Lannoy, G.J.M., Dunbar, R.S., Kim, S.B., Das, N.N., Cosh, M., Walker, J.P., Wagner, W., (2017). Joint Sentinel-1 and SMAP data assimilation to improve soil moisture estimates, Geophys. Res. Lett., 44, 61456153, https://doi.org/10.1002/2017GL073904.

Liu, C., Xue, M., (2016). Relationships among Four-Dimensional Hybrid EnsembleVariational Data Assimilation Algorithms with Full and Approximate Ensemble Covariance Localization. Mon. Wea. Rev., 144, 591606, http://dx.doi.org/10.1175/MWR-D-15-0203.1.

Luo, L., Wood, E.F., Pan, M., (2007). Bayesian merging of multiple climate model forecasts for seasonal hydrological predictions. Journal of Geophysical Research, 112, D10102. http://dx.doi.org/10.1029/2006JD007655.

Massoud, E. C., Huisman, J. , Beninc, E. , Dietze, M. C., Bouten, W. , Vrugt, J. A., Adler, F., (2018). Probing the limits of predictability: data assimilation of chaotic dynamics in complex food webs. Ecol Lett, 21: 93-103, http://dx.doi.org/10.1111/ele.12876.

Mayer-Gürr, T., Zehentner, N., Klinger, B., Kvas, A., (2014). ITSG-Grace2014: a new GRACE gravity field release computed in Graz. - in: GRACE Science Team Meeting (GSTM), Potsdam am: 29.09.2014.

Miralles, D.G., Holmes, T.R.H., de Jeu, R.A.M., Gash, J.H., Meesters, A.G.C.A., Dolman, A.J., (2011), Global land-surface evaporation estimated from satellite-based observations, Hydrology and Earth System Sciences, 15, 453469.

Mu, Q., Heinsch, F.A., Zhao, M., Running, S.W., (2007). Development of a global evapotranspiration algorithm based on MODIS and global meteorology data. Remote Sensing of Environment 111, 519-536, http://dx.doi.org/10.1016/j.rse.2007.04.015.

Munier, S., Aires, F., Schlaffer, S., Prigent, C., Papa, F., et al., (2015). Combining datasets of satellite retrieved products for basin-scale water balance study. Part II: Evaluation on the Mississippi Basin and closure correction model. Journal of Geophysical Research: Atmospheres, American Geophysical Union, 2014, 119, pp.100-116.

Njoku, E.G. et al. (2003). Soil moisture retreival from AMSR-e. IEEE Transactions on Geo-science and Remote Sensing. 41:2, 215-229. 
Njoku, E.G., (2004). AMSR-E/Aqua Daily L3 Surface Soil Moisture, Interpretive Parameters, QC EASE-Grids. Version 2. [indicate subset used]. Boulder, Colorado USA: NASA National Snow and Ice Data Center Distributed Active Archive Center. doi: 10.5067/AMSR-E/AE_LAND3.002.

Pan, M., Wood, E.F., (2006). Data assimilation for estimating the terrestrial water budget using a constrained ensemble Kalman filter. Journal of Hydrometeorology, 7 (3), 534547.

Pan, M., Sahoo, A.K., Troy, T.J., Vinukollu, R.K., Sheffield, J., Wood, E.F., (2012). Multisource Estimation of Long-Term Terrestrial Water Budget for Major Global River Basins. Journal of Climate, 25 (9), 31913206.

Reager, J.T., Thomas, A.C., Sproles, E.A., Rodell, M., Beaudoing, H.K., Li, B., Famiglietti, J.S., (2015). Assimilation of GRACE Terrestrial Water Storage Observations into a Land Surface Model for the Assessment of Regional Flood Potential. Remote Sensing, 7(11):14663-14679, doi:10.3390/rs71114663.

Reichle, R.H., Koster, R.D., (2004). Bias reduction in short records of satellite soil moisture, Geophys. Res. Lett., 31, L19501, http://dx.doi.org/10.1029/2004GL020938.

Renzullo, L.J., Van Dijk, A.I.J.M., Perraud, J.M., Collins, D., Henderson, B., Jin, H., Smith, A.B., McJannet, D.L., (2014). Continental satellite soil moisture data assimilation improves root-zone moisture analysis for water resources assessment. J. Hydrol., 519, 27472762. http://dx.doi.org/10.1016/j.jhydrol.2014.08.008.

Rodell, M., Chen, J., Kato, H., Famiglietti, J.S., Nigro, J., Wilson, C.R., (2007). Estimating groundwater storage changes in the Mississippi River basin (USA) using GRACE, Hydrogeol. J., $15,159166$.

Sahoo, A.K., Pan, M., Troy, T.J., Vinukollu, R.K., Sheffield, J., Wood, E.F., (2011). Reconciling the global terrestrial water budget using satellite remote sensing. Remote Sensing of Environment, 115 (8), 1850-1865.

Sato, M., (2001). Online model selection based on the variational Bayes. Neural Computation, 13, $1649-1681$.

Schneider, U., Fuchs, T., Meyer-Christoffer, A., Rudolf, B., (2008). In G. P. C. Centre (Ed.), Internet publication. 
Seoane, L., Ramillien, G., Frappart, F., Leblanc, M., (2013). Regional GRACE-based estimates of water mass variations over Australia: validation and interpretation, Hydrol. Earth Syst. Sci., 17, 4925-4939, http://dx.doi.org/10.5194/hess-17-4925-2013.

Schumacher, M., Kusche, J., Dll, P., (2016). A systematic impact assessment of GRACE error correlation on data assimilation in hydrological models, Journal of Geodesy, http://dx.doi.org/10.1007/s00190-016-0892-y.

Simmons, A. J., Uppala, S., Dee, D., Kobayashi, S., (2007). ERA-interim: New ECMWF reanalysis products from 1989 onwards, ECMWF Newsletter No. 110 Winter 2006/07.

Simon, D., Chia, T.L., (2002). Kalman filtering with state equality constraints. IEEE Trans. Aerosp. Electron. Syst., 38, 128136.

Smidl, V., Quinn, A., (2006). The Variational Bayes Method in Signal Processing. Springer.

Smidl, V., Quinn, A., (2008). Variational Bayesian Filtering. IEEE Transactions on Signal Processing, 56, 5020-5030.

Smith, A.B., Walker, J.P., Western, A.W., Young, R.I., Ellett, K.M., Pipunic, R.C., Richter, H., (2012). The Murrumbidgee soil moisture monitoring network data set. Water Resour. Res. 48 (7), 16. http://dx.doi.org/10.1029/2012WR011976.

Sokolov, A.A., Chapman, T.G., (1974). Methods for Water Balance Computation An International Guide for Research and Practice. The Unesco Press, Paris.

Strassberg, G., Scanlon, B.R., Rodell, M., (2007). Comparison of seasonal terrestrial water storage variations from GRACE with groundwater-level measurements from the High Plains Aquifer (USA), Geophys. Res. Lett., 34, L14402, http://dx.doi.org/10.1029/2007GL030139.

Su, C.-H., Ryu, D., Young, R.I., Western, A.W., Wagner, W., (2013). Inter-comparison of microwave satellite soil moisture retrievals over the Murrumbidgee Basin, southeast Australia. Remote Sensing of Environment, 134, 111.

Swenson, S., Chambers, D., Wahr, J., (2008). Estimating geocentervariations from a combination of GRACE and ocean model output. Journal of Geophysical research, 113, B08410, http://dx.doi.org/10.1029/2007JB005338. 
Tangdamrongsub, N., Steele-Dunne, S.C., Gunter, B.C., Ditmar, P.G., Sutanudjaja, E.H., Xie, T, Wang, Z., (2017). Improving estimates of water resources in a semi-arid region by assimilating GRACE data into the PCR-GLOBWB hydrological model, Hydrology and Earth System Sciences, 21, 2053-2074.

Tian, S., Tregoning, P., Renzullo, L.J., van Dijk, A.I.J.M., Walker, J.P., Pauwels, V.R.N., Allgeyer, S., (2017). Improved water balance component estimates through joint assimilation of GRACE water storage and SMOS soil moisture retrievals, Water Resour. Res., 53, http://dx.doi.org/10.1002/2016WR019641.

Tregoning, P., McClusky, S., van Dijk, A.I.J.M., Crosbie, R.S., Pea-Arancibia, J.L., (2012). Assessment of GRACE Satellites for Groundwater Estimation in Australia, National Water Commission, Canberra, 82 pp.

van Dijk, A.I.J.M., (2010). The Australian Water Resources Assessment System: Technical Report 3, Landscape model (version 0.5) Technical Description, CSIRO: Water for a Healthy Country National Research Flagship.

van Dijk, A.I.J.M., Renzullo, L.J., Wada, Y., Tregoning, P., (2014). A global water cycle reanalysis (20032012) merging satellite gravimetry and altimetry observations with a hydrological multimodel ensemble. Hydrol Earth Syst Sci 18:29552973. http://dx.doi.org/10.5194/hess-18-29552014.

Wahr, J.M., Molenaar, M., Bryan, F., (1998). Time variability of the Earth's gravity field: hydrological and oceanic effects and their possible detection using GRACE. J Geophys Res 108(B12):3020530229, http://dx.doi.org/10.1029/98JB02844.

Zaitchik, B.F., Rodell, M., Reichle, R.H., (2008). Assimilation of GRACE terrestrial water storage data into a land surface model: results for the Mississippi River Basin. J Hydrometeorol 9(3):535548, http://dx.doi.org/10.1175/2007JHM951.1.

Zhang, J., Campbell, J.R., Hyer, E.J., Reid, J.S.,Westphal, D.L., Johnson R.S., (2014). Evaluating the impact of multisensor data assimilation on a global aerosol particle transport model, J. Geophys. Res. Atmos., 119, 46744689, http://dx.doi.org/10.1002/2013JD020975.

Zhang, Y., Pan, M., Wood, E.F., (2016). On Creating Global Gridded Terrestrial Water Budget Estimates from Satellite Remote Sensing. Surveys in Geophysics, 37 (2), 249268. 


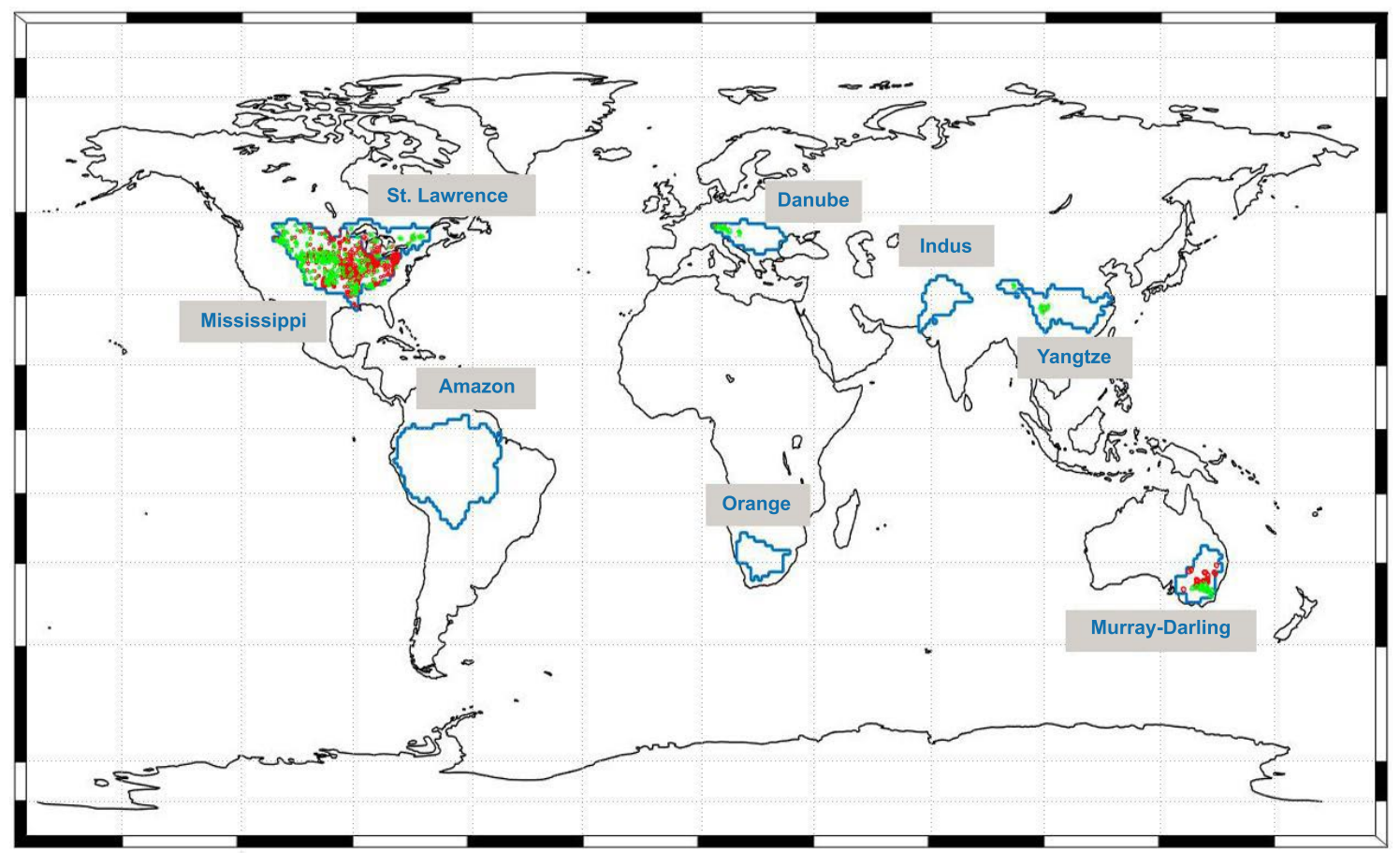

Figure 1: The location of study basins. The figure also contains the distribution of in-situ groundwater (red) and soil moisture (green) gauge stations. 


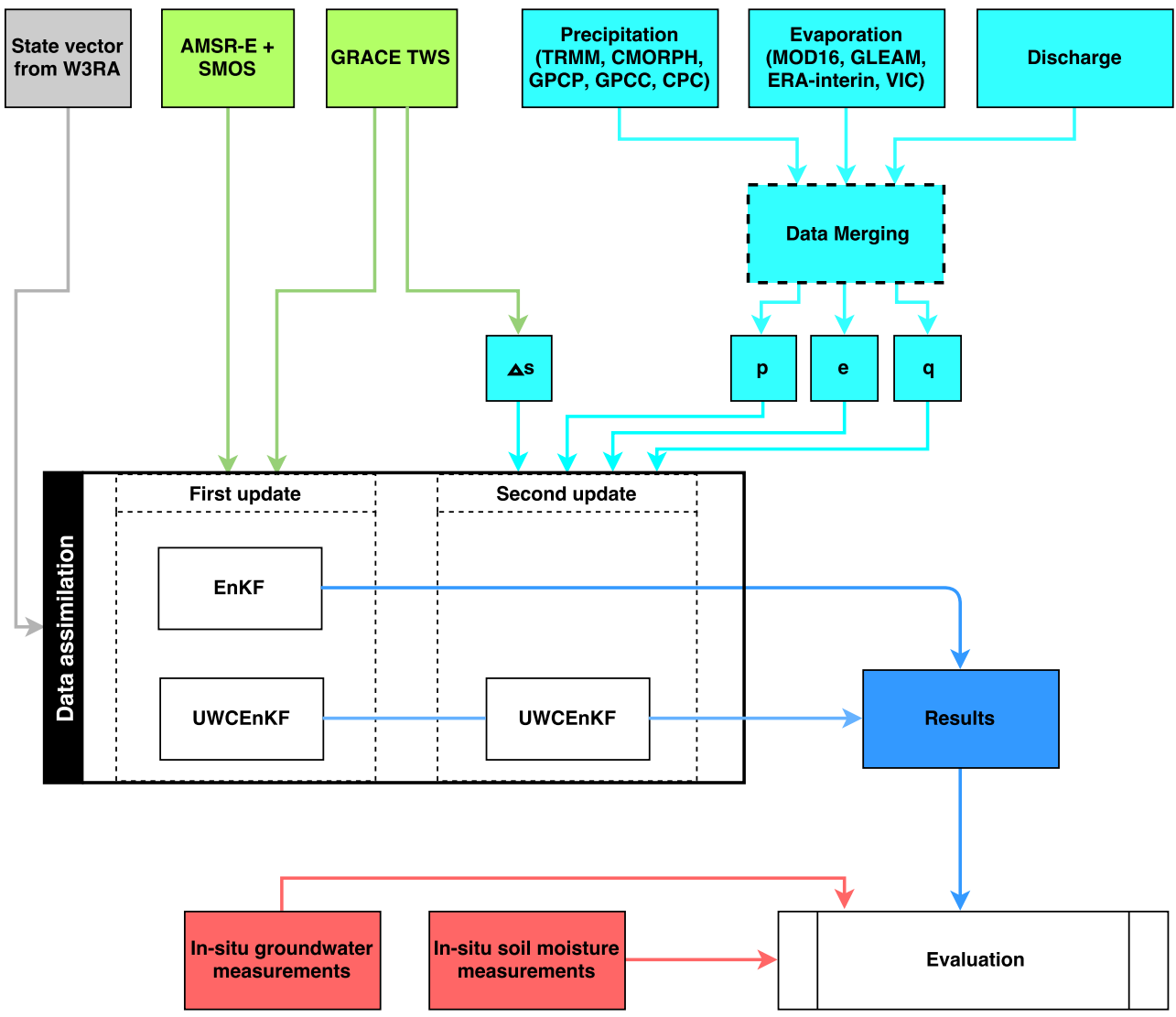

Figure 2: A schematic illustration of the UWCEnKF steps applied for data assimilation, as well as data merging process. 


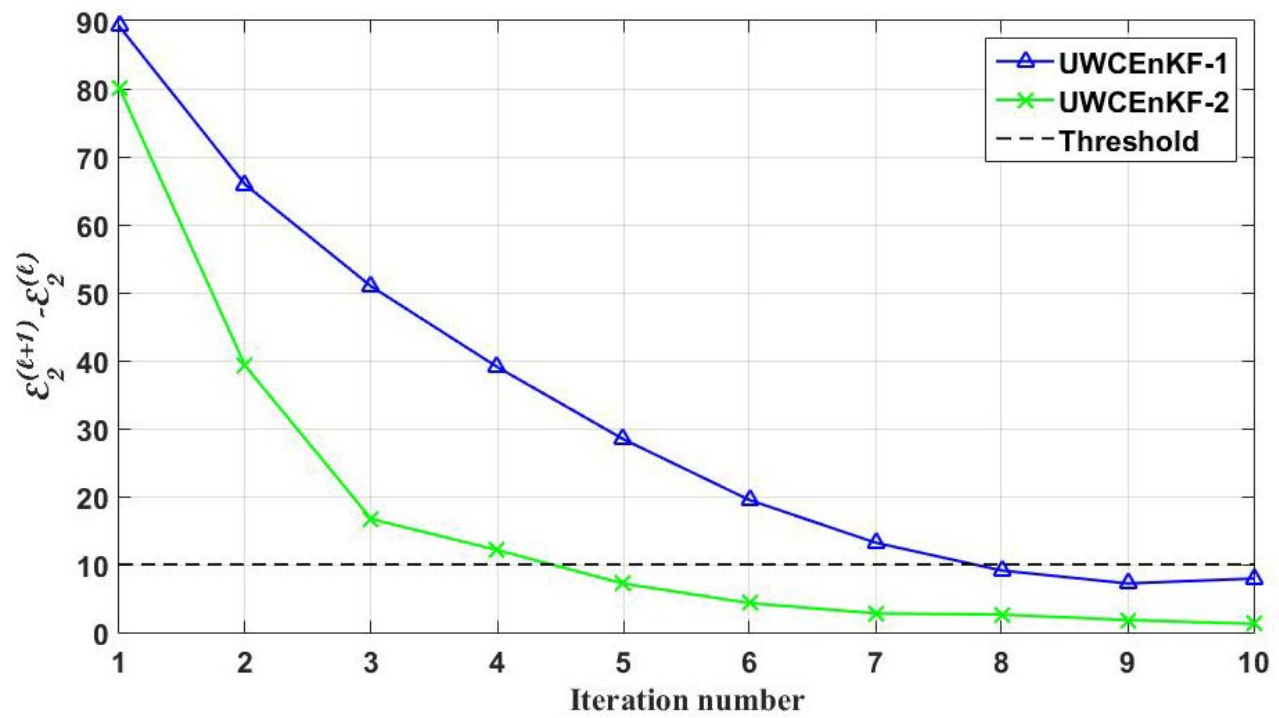

Figure 3: Average $\mathcal{E}_{2}^{(\ell+1)}-\mathcal{E}_{2}^{(\ell)}$ estimates (unit is mm) from UWCEnKF-1 and UWCEnKF-2 filters during assimilation in each iteration (for $\ell=0 \cdots 10$ ). The threshold value $(10 \mathrm{~mm}$ ) is chosen arbitrary based on a trial and error procedure. 


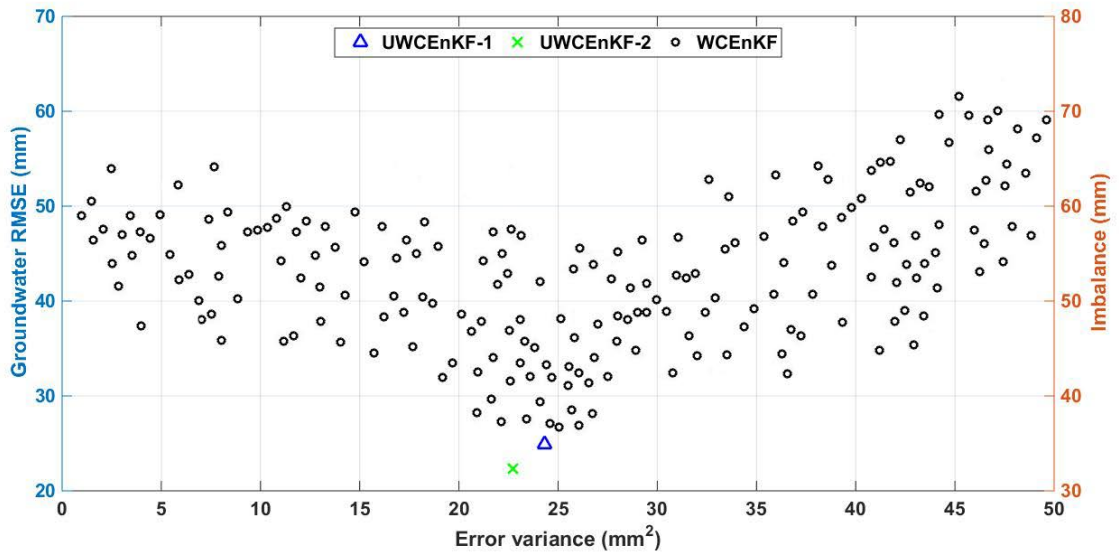

Figure 4: Average groundwater RMSE and imbalance for various implementations of the WCEnKF filter using different error variance assumed (circles) considering different error variance. UWCEnKF-1 and UWCEnKF-2 results are indicated by triangle and cross, respectively. 




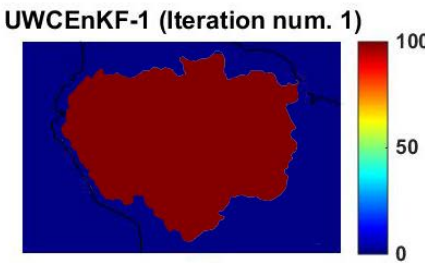

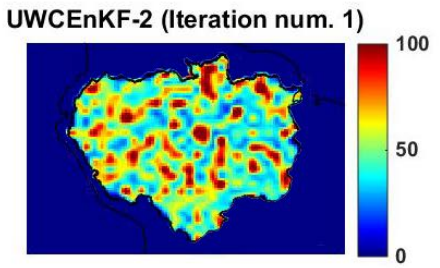

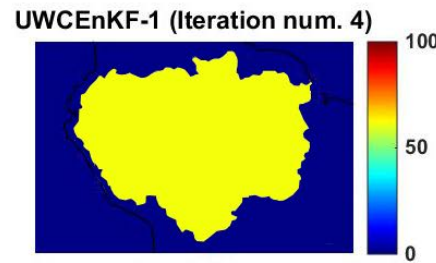

UWCEnKF-2 (Iteration num. 4)

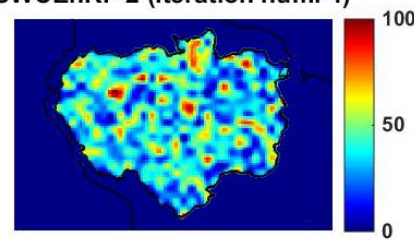

UWCEnKF-1 (Iteration num. 8)

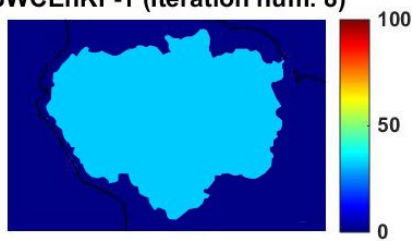

UWCEnKF-2 (Iteration num. 8)

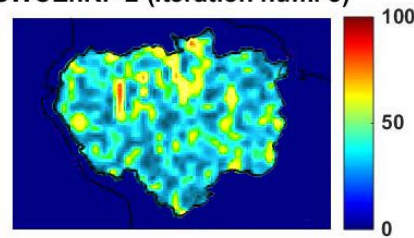

Figure 5: Spatial variability of error variances estimated by WCEnKF, UWCEnKF-1, and UWCEnKF-2. The corresponding results for different iterations are also demonstrated for WCEnKF-1 and UWCEnKF-2. 


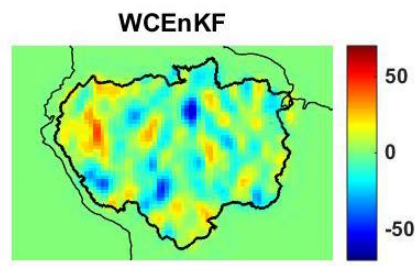

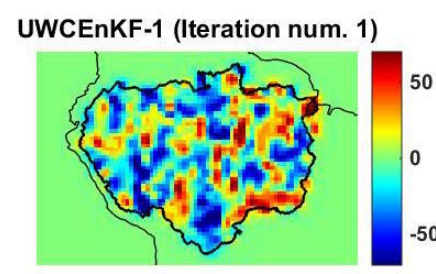

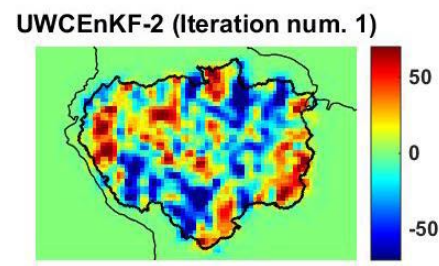

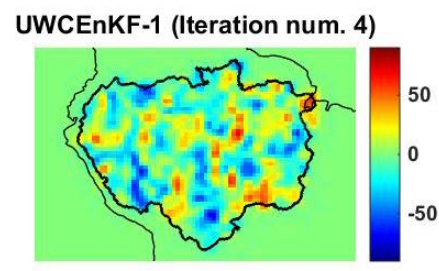



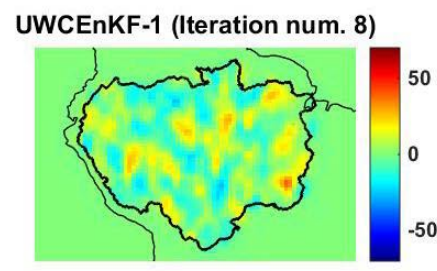

UWCEnKF-2 (Iteration num. 8)

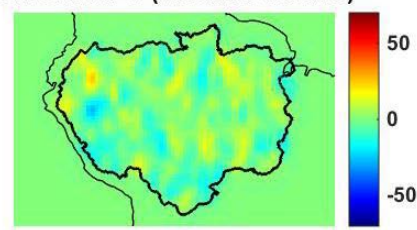

Figure 6: Spatial variability of imbalances from WCEnKF, UWCEnKF-1, and UWCEnKF-2 corresponding to the errors presented in Figure 5. 
(a)

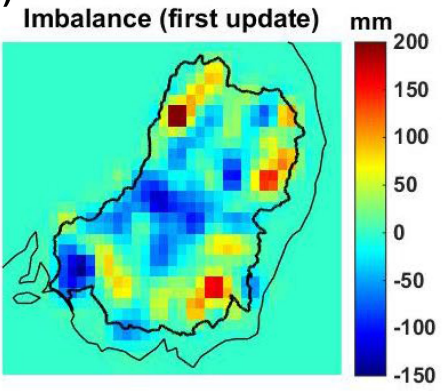

(b)

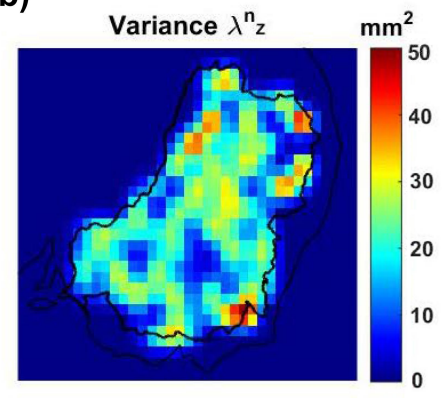

(c)

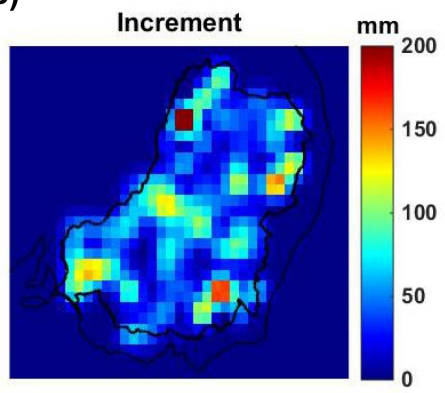

Figure 7: Temporarily averaged maps of imbalances from UWCEnKF-2's first update (a), estimated error variance (b), and increments applied in the second analysis step of UWCEnKF-2 (c). 


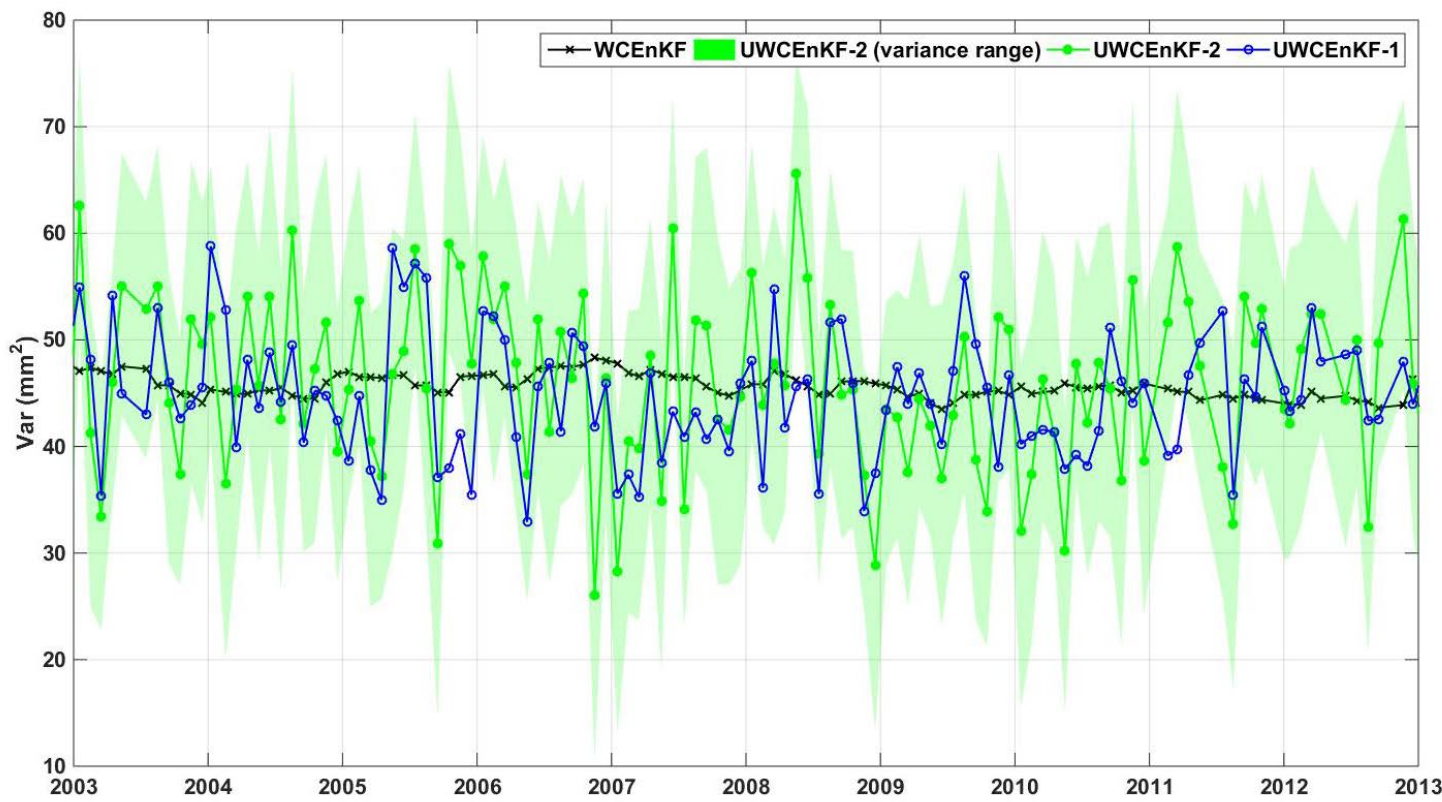

Figure 8: Average water balance variances estimated by UWCEnKF-1 and UWCEnKF-2. The plots also contains the assigned variance values for WCEnKF implementation. 
+ WCEnKF $\triangle$ UWCEnKF-1 $\bigcirc$ UWCEnKF-2

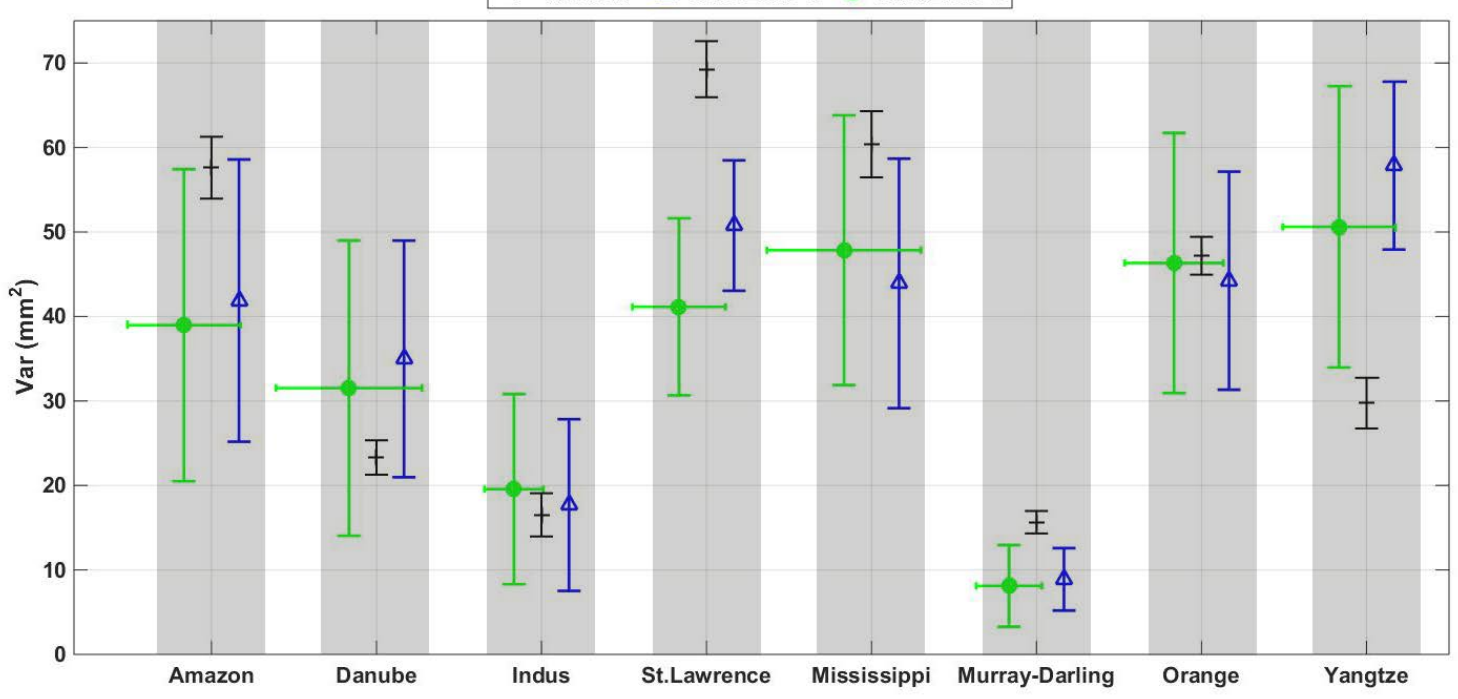

Figure 9: Variation ranges of water balance covariance in time (vertical lines) and space (horizontal lines) for WCEnKF, UWCEnKF-1, and UWCEnKF-2. 


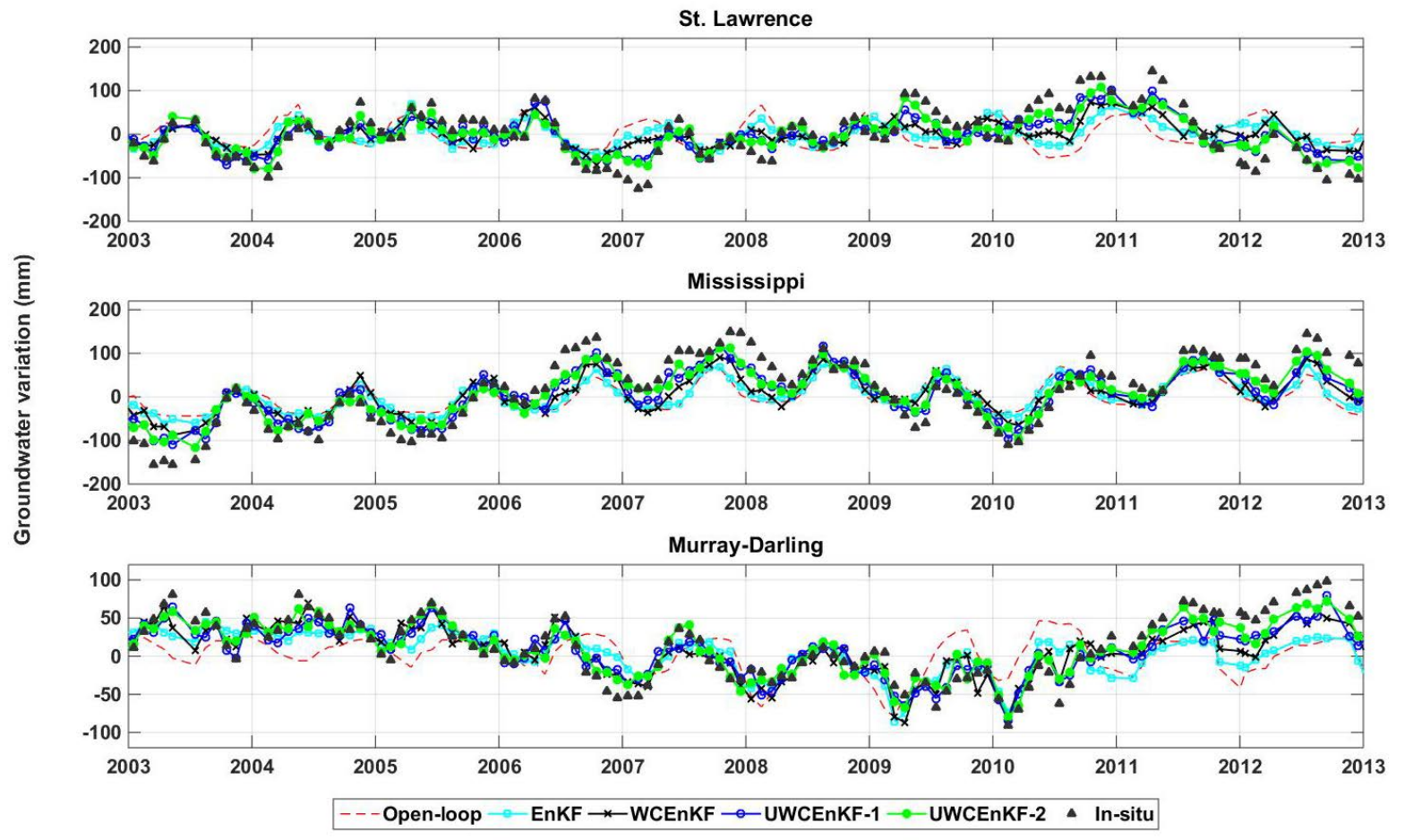

Figure 10: Average groundwater variation time series by the open-loop run, EnKF, WCEnKF, UWCEnKF-1, and UWCEnKF-2 over St. Lawrence, Mississippi, and Murray-Darling basins. 

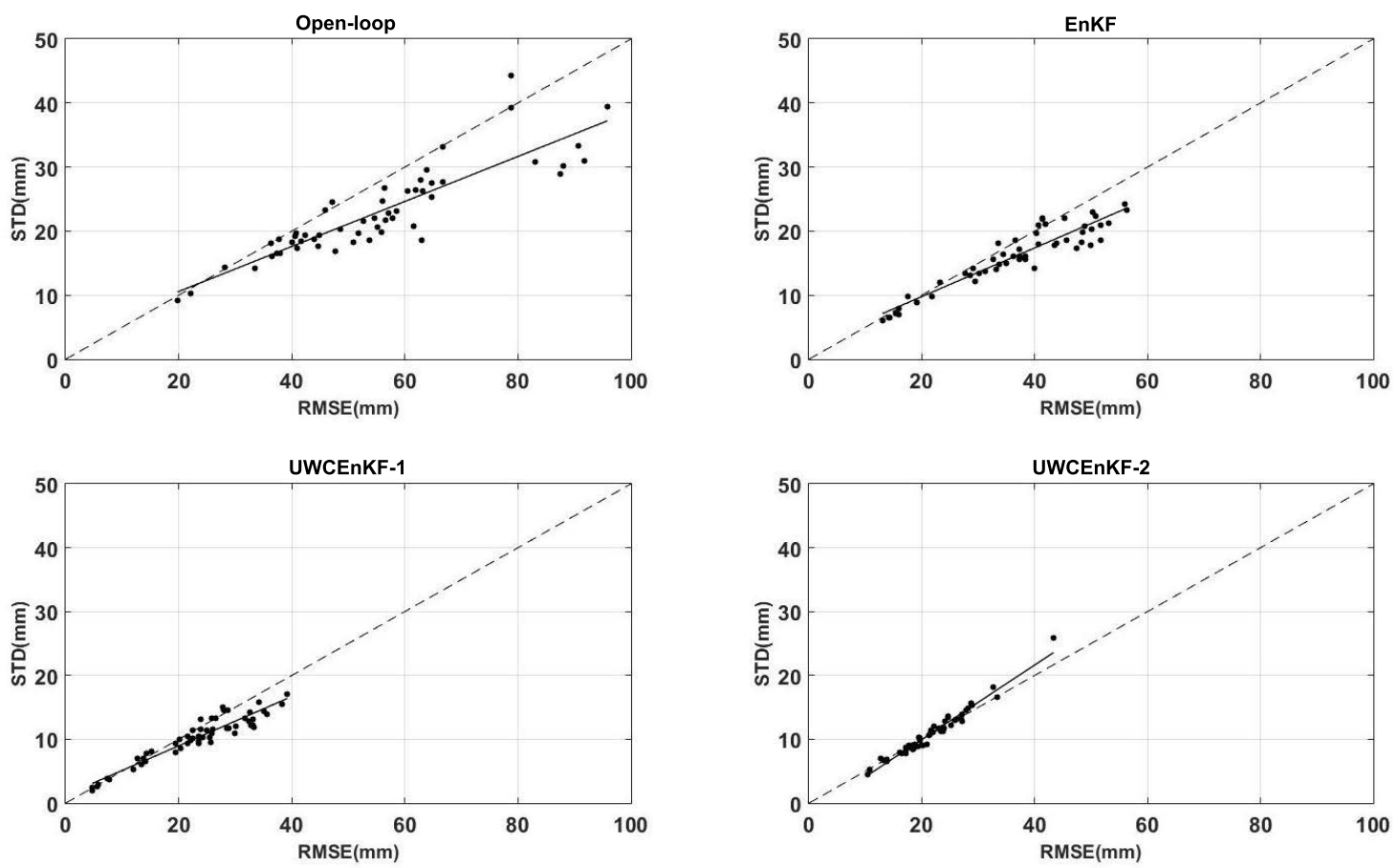

Figure 11: Average RMSE and STD of the groundwater results from the EnKF, UWCEnKF-1, and UWCEnKF-2 filters over the Murray-Darling basin regarding the in-situ groundwater measurements. 

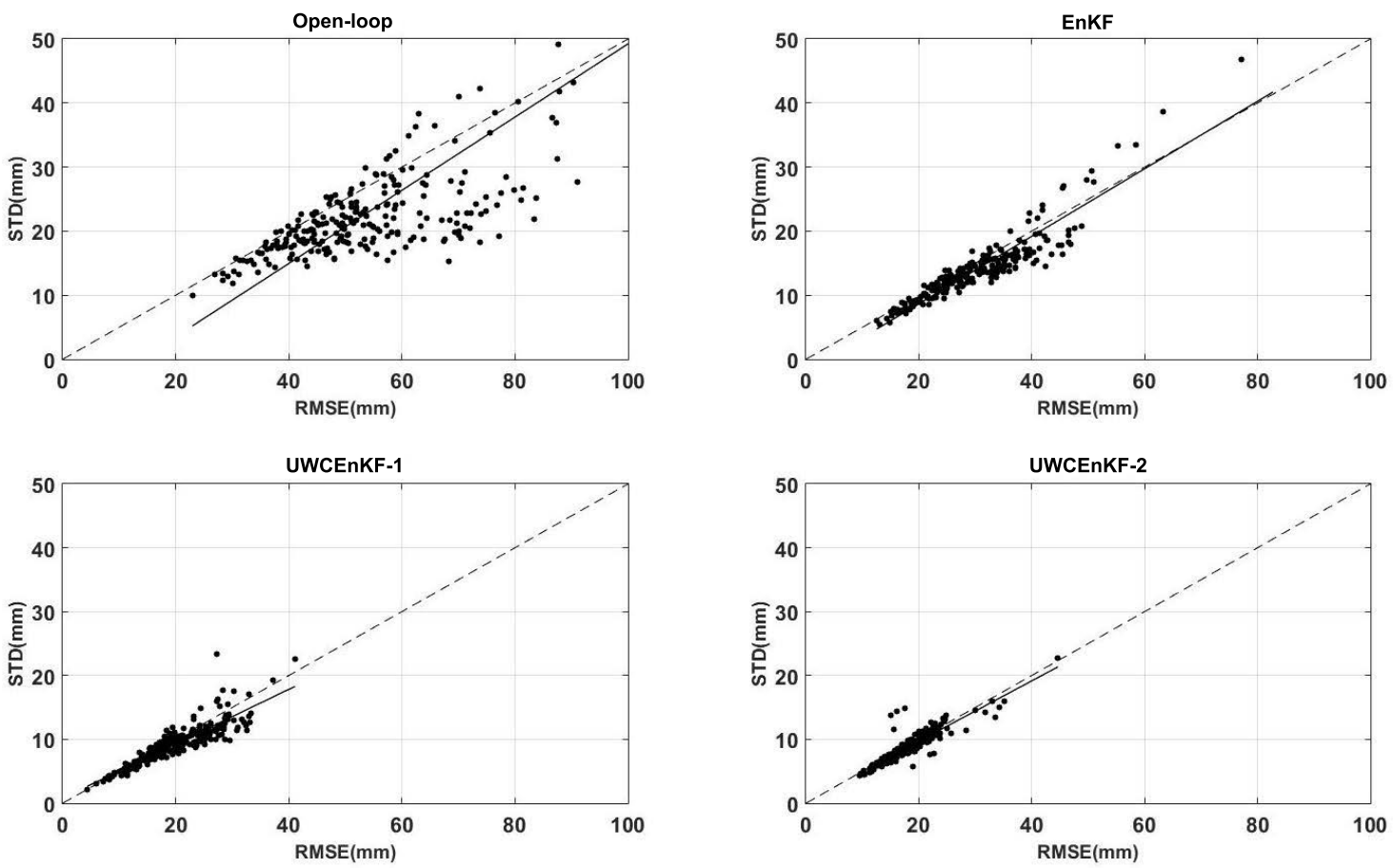

Figure 12: Average RMSE and STD of the groundwater results from the EnKF, UWCEnKF-1, and UWCEnKF-2 filters over the Mississippi basin regarding the in-situ groundwater measurements. 


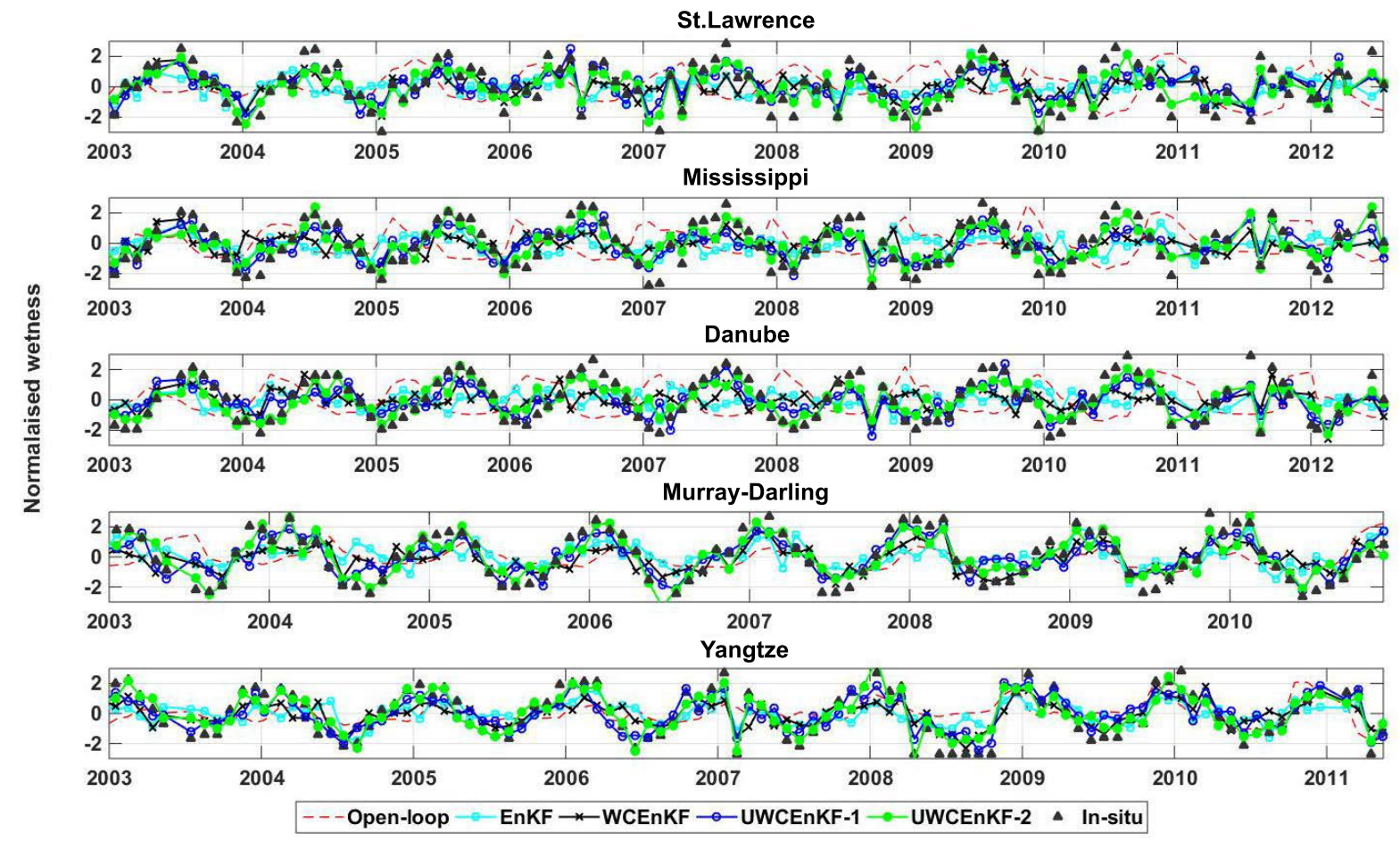

Figure 13: Average soil moisture variation time series by the open-loop run, EnKF, WCEnKF, UWCEnKF-1, and UWCEnKF-2 over St. Lawrence, Mississippi, Danube, Yangtze, and Murray-Darling basins. 

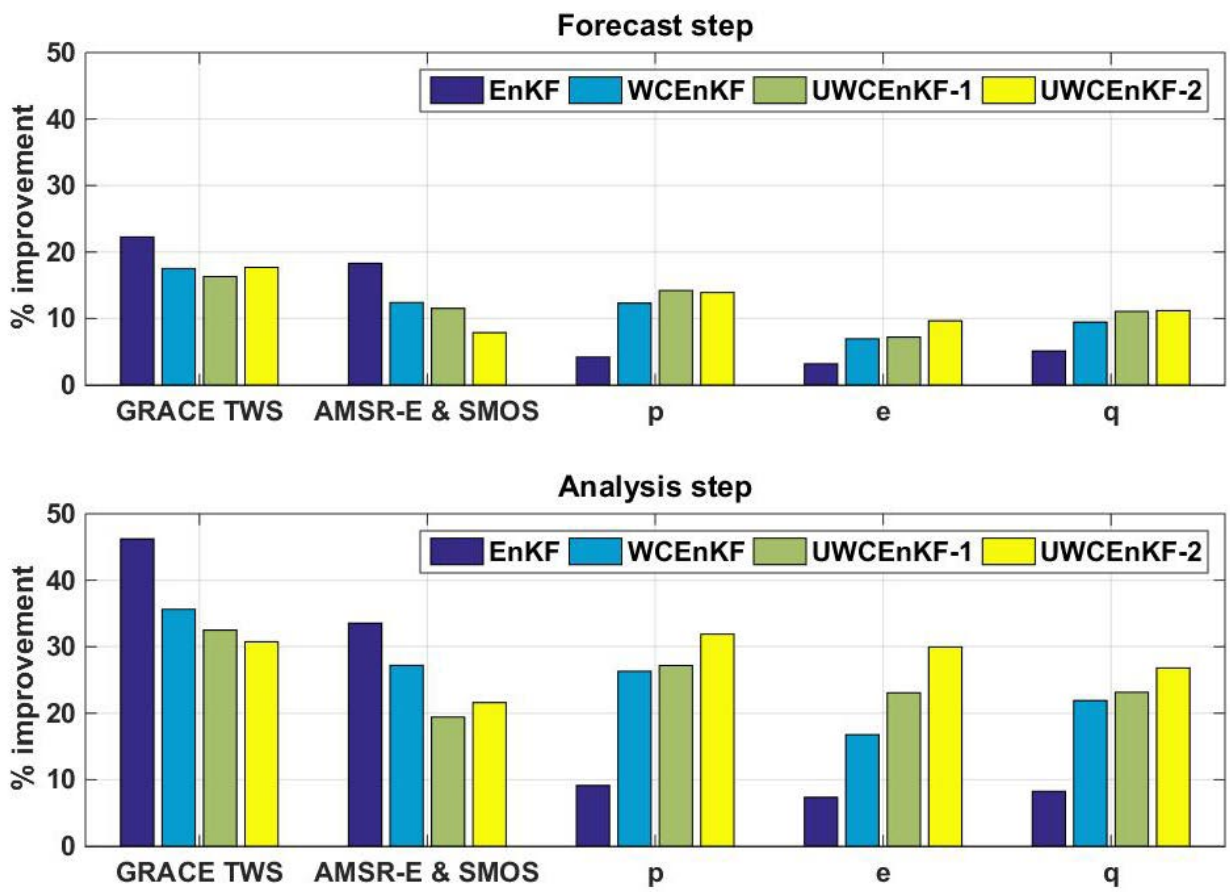

Figure 14: Average correlation improvements of filtered TWS time series to GRACE TWS, $\mathbf{p}$, e, and discharge q with respect to open-loop run in forecast and analysis steps. For AMSR-E+SMOS correlation, filtered top soil storage estimates are used. 

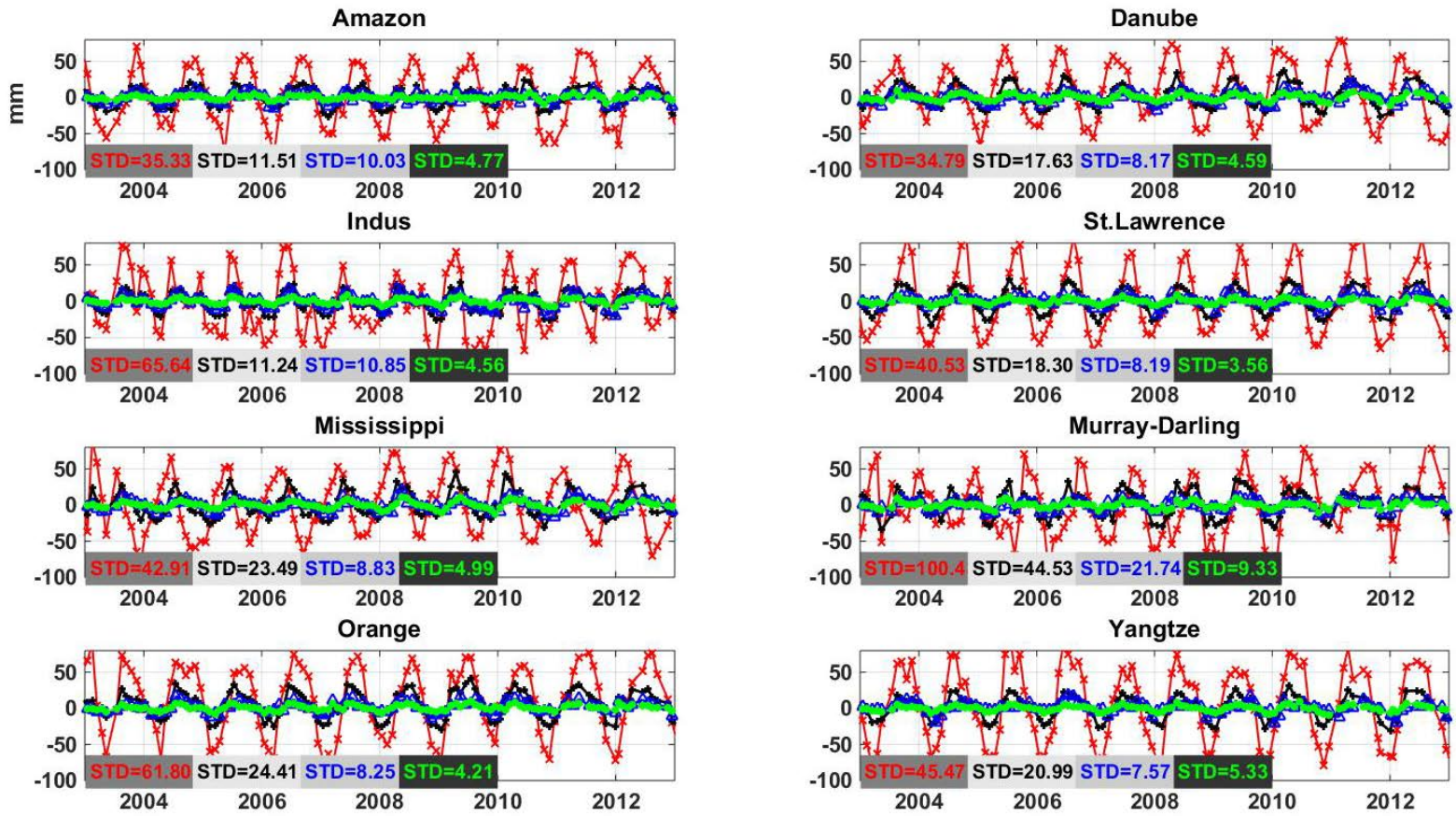

*-EnKF $\longrightarrow$ WCEnKF $\triangle$ UWCEnKF-1 --*-UWCEnKF-2

Figure 15: Average water budget imbalance time series calculated using EnKF, WCEnKF, and UWCEnKF variants for each basin (units are $\mathrm{mm}$ ). 
Table 1: A summary of the datasets used in this study.

\begin{tabular}{|c|c|c|}
\hline Product & Platform & Reference \\
\hline Terrestrial water storage (TWS) & GRACE & Mayer-Gürr et al. (2014) \\
\hline Soil moisture & AMSR-E & Njoku (2004) \\
\hline Soil moisture & SMOS & Draper et al. (2009) \\
\hline Precipitation $(\mathbf{p})$ & TRMM-3B42 & Huffman et al. (2007) \\
\hline Precipitation $(\mathbf{p})$ & CMORPH & Joyce et al. (2004) \\
\hline Precipitation $(\mathbf{p})$ & GPCP & Adler et al. (2003) \\
\hline Precipitation $(\mathbf{p})$ & GPCC & Schneider et al. (2008) \\
\hline Precipitation $(\mathbf{p})$ & $\mathrm{CPC}$ & Chen et al. (2002) \\
\hline Evapotranspiration (e) & MOD16 & Mu et al. (2007) \\
\hline Evapotranspiration (e) & GLEAM & Miralles et al. (2011) \\
\hline Evapotranspiration (e) & ERA-interim & Simmons et al. (2007) \\
\hline Evapotranspiration (e) & VIC & Liang et al. (1994) \\
\hline Water discharge (q) & GRDC & $\begin{array}{l}\text { http://www . bafg.de/GRDC/EN/Home/homepage_node. } \\
\text { html }\end{array}$ \\
\hline Water discharge $(\mathbf{q})$ & & $\begin{array}{l}\text { http://www.hydrosciences.fr/sierem/consultation/ } \\
\text { choixaccess.asp?lang=en }\end{array}$ \\
\hline Water discharge $(\mathbf{q})$ & USGS & https://waterdata.usgs.gov/nwis/sw \\
\hline Water discharge (q) & & http://www.bom.gov.au/waterdata/ \\
\hline Water discharge $(\mathbf{q})$ & NRFA & http://nrfa.ceh.ac.uk/data/ \\
\hline Water discharge (q) & & http://www. ore-hybam.org/ \\
\hline Water discharge $(\mathbf{q})$ & & $\begin{array}{l}\text { http://www .hydrology gov.np/new/bull3/index.php/ } \\
\text { hydrology/home/main }\end{array}$ \\
\hline Hydrological model & W3RA & http://www.wenfo.org/wald/data-software/ \\
\hline Groundwater in-situ measurements & NSW & http://waterinfo.nsw.gov.au/pinneena/gw.shtml \\
\hline Groundwater in-situ measurements & USGS & https://water.usgs.gov/ogw/data.html \\
\hline Soil moisture in-situ measurements & OzNet & Smith et al. (2012) \\
\hline Soil moisture in-situ measurements & ISMN & https://ismn.geo.tuwien.ac.at/ \\
\hline
\end{tabular}


Table 2: Average correlations between in-situ and soil moisture estimates from various methods. Improvements in the assimilation results are calculated as [(assimilation - open-loop run)/open-loop run] $\times 100(\%)$.

\begin{tabular}{cccccc}
\hline Basin & Open-loop & EnKF & WCEnKF & UWCEnKF-1 & UWCEnKF-2 \\
\hline Danube & 0.67 & 0.74 & 0.79 & 0.81 & 0.82 \\
St. Lawrence & 0.69 & 0.72 & 0.76 & 0.84 & 0.87 \\
Mississippi & 0.72 & 0.81 & 0.85 & 0.86 & 0.88 \\
Murray-Darling & 0.76 & 0.83 & 0.86 & 0.89 & 0.91 \\
Yangtze & 0.73 & 0.75 & 0.78 & 0.80 & 0.81 \\
\hline Improvemts (\%) & - & 7.85 & 13.22 & 17.75 & 20.28 \\
\hline
\end{tabular}


Table 3: Average correlations between the filtered water discharge and independent observations over different basins.

\begin{tabular}{ccccc}
\hline Basin & Open-loop & EnKF & UWCEnKF-1 & UWCEnKF-2 \\
Amazon & 73.62 & 78.04 & 95.26 & 96.58 \\
Danube & 76.13 & 76.28 & 90.77 & 90.60 \\
Indus & 77.08 & 74.71 & 84.48 & 85.37 \\
St. Lawrence & 68.55 & 80.65 & 87.41 & 89.17 \\
Mississippi & 71.91 & 73.78 & 94.29 & 93.32 \\
Murray-Darling & 79.36 & 83.12 & 96.31 & 96.89 \\
Orange & 69.47 & 71.82 & 93.42 & 94.05 \\
Yangtze & 71.15 & 75.49 & 92.69 & 93.91 \\
\hline
\end{tabular}

